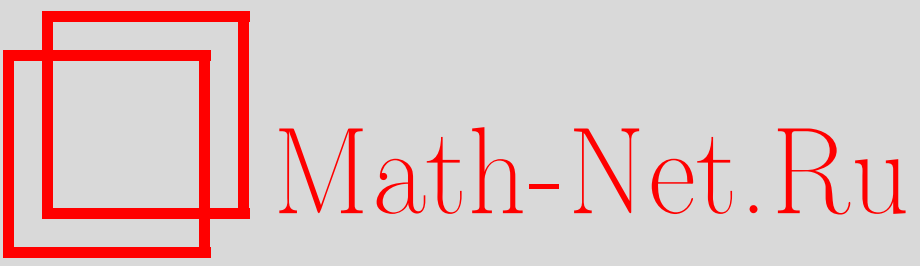

А. С. Горский, Интегрируемые многочастичные системы и калибровочные теории, ТМФ, 2000, том 125, номер 1, 3-56

DOI: https://doi.org/10.4213/tmf656

Использование Общероссийского математического портала Math-Net.Ru подразумевает, что вы прочитали и согласны с пользовательским соглашением

http://www.mathnet.ru/rus/agreement

Параметры загрузки:

IP: 54.198 .55 .26

26 апреля 2023 г., 15:10:24

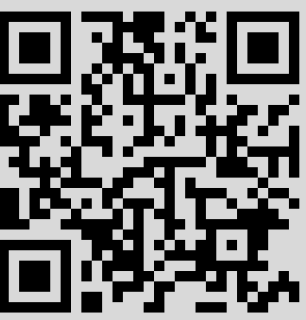




\section{ИНТЕГРИРУЕМЫЕ МНОГОЧАСТИЧНЫЕ СИСТЕМЫ И КАЛИБРОВОЧНЫЕ ТЕОРИИ ${ }^{1)}$}

Обзор посвящен изучению связи между многочастичными интегрируемыми системами и калибровочными теориями. Показано, каким образом степени свободы интегрируемых систем связаны с топологическими степенями свободы калибровочных теорий. Описаны связи между семействами интегрируемых систем и $N=2$ суперсимметричными калибровочными теориями. Показано, что степени свободы многочастичных систем могут быть отождествлены с коллективными координатами солитонов в теории струн - D-бран.

\section{СОДЕРЖАНИЕ}

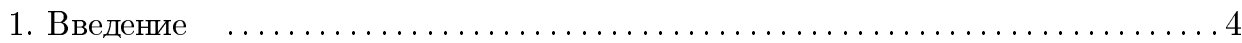

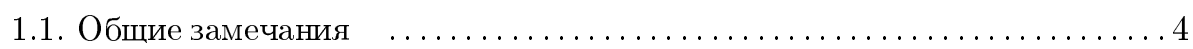

1.2. Модули и непертурбативные степени свободы $\quad \ldots \ldots \ldots \ldots \ldots \ldots \ldots . .5$

2. Многочастичные системы и калибровочные теории в двух и трех измере-

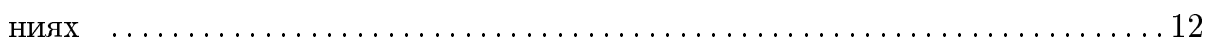

2.1. Многочастичные системы Калоджеро и теория ЯМ в двух измере-

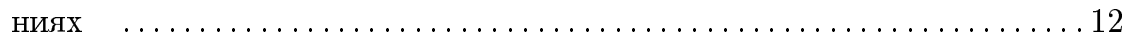

2.2. Системы Рюсенара и калиброванная $G / G \sigma$-модель $\ldots \ldots \ldots \ldots \ldots 15$

2.3. Эллиптическая модель Калоджеро и калибровочные теории . . . . . 19

3. Дуальности в многочастичных системах и калибровочные теории $\ldots \ldots .21$

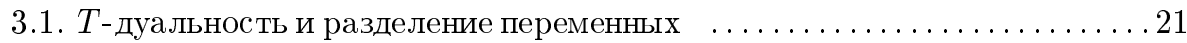

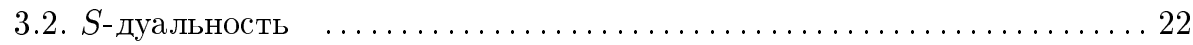

3.3. Зеркальная симметрия в динамических системах $\ldots \ldots \ldots \ldots \ldots \ldots 23$

4. Системы частиц и суперсимметричные калибровочные теории $\ldots \ldots \ldots 28$

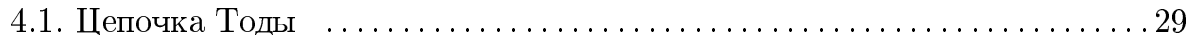

4.2. Теория с гипермультиплетом в присоединенном представлении и систе-

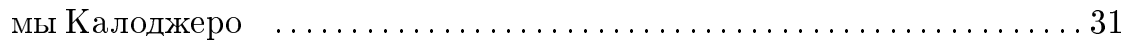

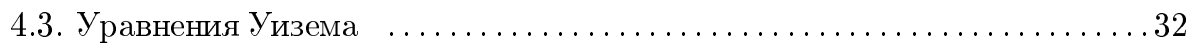

\footnotetext{
${ }^{1)}$ Статья написана по заказу Редколлегии.
}

\section{* Институт теоретической и экспериментальной физики, Москва, Россия}


4.4. Суперсимметричная КХД и $X X X$-спиновые цепочки $\ldots \ldots \ldots \ldots . . . .34$

4.5. Твистованная неоднородная $X X Z$-спиновая цепочка и 5-мерные тео-

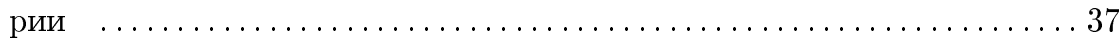

5. Браны как степени свободы при низких энергиях в суперсимметричных ка-

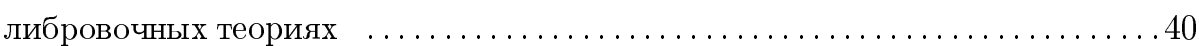

5.1. Теории поля на мировой поверхности D-браны $\ldots \ldots \ldots \ldots \ldots \ldots \ldots$

5.2. Степени свободы многочастичных систем и браны $\ldots \ldots \ldots \ldots \ldots \ldots 4$

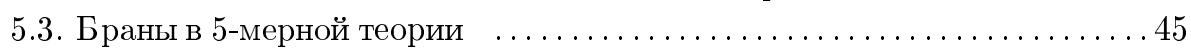

5.4. Уравнения движения в многочастичных системах и браны $\ldots \ldots \ldots 48$

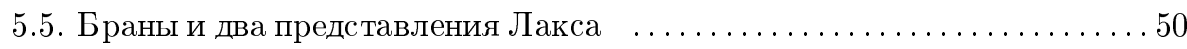

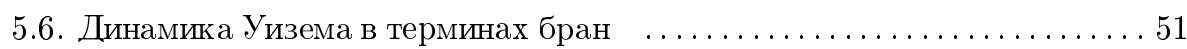

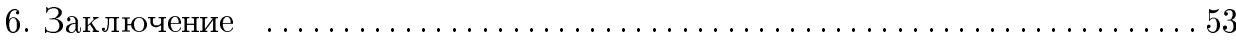

\section{1. ВВЕДЕНИЕ}

1.1. Общие замечания. В обзоре обсуждаются вопросы, связанные со скрытой интегрируемостью систем, в которых на первый взгляд нет необходимого числа законов сохранения. Эта ситуация сильно отличается от привычной для теории интегрируемых систем. Действительно, в обычном случае обсуждается лагранжиан хорошо определенной теории поля, переменными в интегрируемой системе являются поля лагранжиана, а в качестве времени выбирается нормальная временная переменная в двумерном пространстве-времени.

В интересуюших нас системах в качестве координат фазовых пространств в интегрируемой динамике выступают те или иные коллективные координаты или модули, существенные в задаче. Коллективные координаты появляются при рассмотрении многих задач и имеют различное происхождение. Они могут описывать эффективные степени свободы, существенные в том или ином режиме в теориях поля, которые не являются полностью интегрируемыми. Режим может определяться либо кинематикой процесса, либо выбором специальных топологически нетривиальных данных. Если имеется несколько коллективных степеней свободы, то, как правило, в теории имеется скрытая интегрируемость, происхождение которой связано с симметрийным характером пространства модулей. Оказывается, что такой подход работает при описании эффективных действий в 4-мерных суперсимметричных калибровочных теориях, найденных Зайбергом и Виттеном $[1,2]$, и позволяет ввести правильные степени свободы в вакуумном секторе теорий с сильным взаимодействием.

Роль подобной интегрируемости представляется двоякой: она позволяет, во-первых, определить все скрытые симметрии задачи, а во-вторых, выделить правильные степени свободы. В дальнейшем мы покажем, как низкоэнергетический сектор суперсимметричной калибровочной теории описывается конечномерной интегрируемой системой.

Ограниченный объем обзора не позволяет подробно описать необходимые сведения из теории интегрируемых систем, суперсимметричных калибровочных теорий и непертурбативной теории струн. Проблема связана с тем, что вопросы интегрируемости оказы- 
ваются на пересечении слабо связанных между собой разделов теоретической физики, используюших сушественно различаюшиеся методы. В связи с этим можно рекомендовать ряд обзоров, затрагиваюших рассматриваемые вопросы и методы, используемые в дальнейшем, где могут быть найдены все необходимые ссылки на оригинальные работы. В качестве обзора по методу конечнозонного интегрирования в теории интегрируемых систем упомянем работу [3], а по иерархиям Уизема - [4], теория Виттена-Зайберга изложена в [5], а необходимая информация по непертурбативной теории струн может быть найдена в [6]. Сведения из алгебраической геометрии, используемые при изучении бранных конфигураций, описаны в [7], а взгляд на интегрируемость в непертурбативной области теории поля обсуждается в $[8,9]$.

1.2. Модули и непертурбативные степени свободы. Описание области сильной связи остается одной из ключевых задач квантовой теории поля. Так как методы теории возмушений в области сильной связи не работают, основная надежда связана с обнаружением новых эффективных степеней свободы, в терминах которых можно будет развивать теорию возмущений по другим параметрам, отличным от константы связи в исходной теории. Наиболее ярким примером существования неэквивалентных теорий поля на разных масштабах энергии является квантовая хромодинамика (КХД). При высоких энергиях теория описывается в терминах кварков и глюонов, в то время как при низких энергиях применим киральный лагранжиан, определяющий эффективное низкоэнергетическое действие. Степенями свободы в киральном лагранжиане являются поля бесцветных мезонов и барионов, а параметры низкоэнергетического действия не могут быть определены из обших принщипов и фиксируются феноменологически.

Общая структура низкоэнергетических действий определяется принципами симметрии. Ожидается, что эффективные действия наследуют полный набор тождеств Уорда из исходной теории поля. Часть тождеств Уорда аномальна, однако хорошо известно, что аномалии, будучи связанными с явлением пересечения уровней, не перенормируются и допускают эквивалентное описание в инфракрасных и ультрафиолетовых терминах [10]. В силу этого обстоятельства выполнение аномальных тождеств Уорда также накладывается в качестве требования к эффективной теории. В частности, аномальные тождества Уорда, связанные с киральной симметрией, фиксируют лагранжиан мезонов, а тождества Уорда для конформной симметрии - дилатонный эффективньй лагранжиан в обычной и $N=1$ суперсимметричной КХД $[11,12]$.

Одной из особенностей эффективных действий является их универсальность, т.е. свойство нескольких различных ультрафиолетовых теорий порождать одну и ту же теорию в инфракрасной области. Причиной подобной универсальности является жесткость конструкции эффективных действий, фиксируемых симметриями задачи. Более того, симметрия эффективных действий может оказаться выше симметрий исходных ультрафиолетовых теорий. Симметрийньй характер эффективных действий приводит к ряду нетривиальных следствий, из которых наиболее сушественным, пожалуй, является связь с теорией интегрируемых систем. Как правило, статистическая сумма, вычисленная в эффективной теории, оказывается так называемой $\tau$-функцией некоторой интегрируемой системы. Последняя является производяшей функцией для законов со- 
хранения в интегрируемой системе и одновременно производяшей функцией для корреляторов в теории поля [8].

Идентификация переменных в интегрируемой системе, описывающей эффективное действие, представляет собой непростую задачу. На настояший момент инвариантный способ введения переменных в теориях, которые не являются топологическими, отсутствует, однако имеется ряд примеров в двумерных теориях, в которых в качестве переменных интегрируемых систем выступают амплитуды перехода между различными вакуумами теории, индуцированного непертурбативными конфигурациями. Роль “пространственно-временных переменных" интегрируемых систем играют константы связи и источники в теории поля. Примеры систем, связанных с иерархиями двумеризованной цепочки Тоды и Кортевега-де Фриза $(\mathrm{K} д \Phi)$, могут быть получены в двумерных $\sigma$-моделях [13] и двумерной теории гравитации $[14,15,9,8]$. Отдельно стоит задача выбора конкретных решений нелинейных уравнений в интегрируемой динамике, однако эта проблема, по-видимому, решается, если учесть набор тождеств Уорда, наложенных на теорию [16].

Несмотря на возможность фиксации общей структуры эффективных действий из симметрийных соображений, представляет интерес их непосредственное вычисление интегрированием по “тяжелым" модам в исходной теории поля. Предполагается введение некоторого масштаба энергии, выше которого все моды суммируются. Непосредственное интегрирование ведется по пертурбативным и непертурбативным фолуктуациям, причем суммирование по пертурбативным модам предполагает вычисление диаграмм Фейнмана, для которых введенный масштаб энергии служит обрезанием. В теориях разных размерностей имеются непертурбативные конфигурации, которые также необходимо просуммировать, предполагая, что их характерные размеры ограничены на некотором масштабе. Непертурбативными флуктуациями, существенными в разных размерностях, являются инстантоны в 4-мерной теории поля, монополи, вихри и солитоны разных коразмерностей в теории струн.

Ключевым обстоятельством, в значительной степени определяюшим описание непертурбативных эффектов, является наличие коллективных координат решений, или модулей. Происхождение модулей тесно связано с наличием в исходной теории повышенной симметрии, поэтому пространство модулей само по себе обладает нетривиальными симметрийными свойствами. Простейшим примером может служить пространство модулей инстантонов, размерность которого задается симметриями пространства-временитрансляциями и поворотами, а также калибровочными симметриями $[17,18]$. Другими интересными пространствами модулей являются пространства модулей комплексных структур римановых поверхностей, модули плоских связностей на фиксированной двумерной поверхности, модули голоморфных векторных расслоений на поверхностях. Каждое из этих пространств возникает при описании тех или иных непертурбативных конфигураций и может быть получено процедурой редукции из пространства модулей инстантонов, поэтому универсальное пространство модулей произвольного числа инстантонов является наиболее общим объектом, возникающим при описании теорий поля в размерности $d \leqslant 4$. 
Интегрирование по пространству модулей, неизбежно возникающее при непосредственном получении эффективных действий, приводит к появлению интегрируемых систем и с другой точки зрения. Дело в том, что фазовое пространство интегрируемых систем всегда совпадает с тем или иным пространством модулей или кокасательным расслоением к пространству модулей. В качестве примера можно упомянуть иерархию КдФ, связанную с пространством модулей комплексных структур, или двумеризованную цепочку Тоды, связанную с модулями плоских связностей. В частности, лагранжиан Черна-Саймонса (ЧС), определенный на некоторой поверхности, имеет в качестве фазового пространства пространство модулей плоских связностей на этой поверхности $[19,20]$, а в качестве гамильтонианов могут быть выбраны любые калибровочно-инвариантные наблюдаемые. В терминах интегрируемых систем задача о вычислении вклада непертурбативных флуктуаций в эффективное действие сводится к вычислению средних от некоторых наблюдаемых в интегрируемых системах на пространстве модулей $[20,21]$.

Большой класс теорий, связанных с пространством коллективных координат, носит название топологических теорий поля. Они были введены в квантовую теорию поля Виттеном [22] с целью выделения инвариантов вакуумных многообразий теорий поля. Первые примеры топологических теорий были построены для $\sigma$-моделей и 4 -мерных калибровочных теорий, причем в первом случае топологические теории генерировали инварианты голоморфных отображений, а в 4-мерном случае они определяли инварианты пространства модулей инстантонов [23]. На настоящий момент существует достаточно много примеров топологических теорий в разных размерностях, например топологические $\sigma$-модели в двух, теории ЧС в трех и топологические лагранжианы в четырех измерениях.

Во всех случаях топологические теории могут быть получены процедурой твистования нетопологических теорий с высокой (как правило, $N=2$ ) суперсиммметрией. Статистическая сумма в топологических теориях является инвариантом соответствующего пространства модулей. В ряде примеров в теориях поля сушествуют топологические секторы, которые связаны только с вакуумными состояниями. Наиболее ярким примером является $N=1$ теория, для которой оказывается возможным вычислить точную пертурбативную $\beta$-функцию [24] и некоторые корреляторы [25]. На топологичность низкоэнергетического действия в случае $N=2$ указывает наличие уравнений, аналогичных уравнениям в топологических теориях [26].

Так как часто в приложениях возникают конечномерные пространства модулей, то наиболее интересны топологические теории, определяющие конечномерные фазовые пространства. Именно на фазовых пространствах топологических теорий рассматривается динамика интегрируемых многочастичных систем с нетривиальными законами сохранения. В качестве гамильтонианов выбираются инвариантные наблюдаемые со своими константами связи. Наиболее информативным объектом оказывается статистическая сумма возмущенной топологической теории, которая является производящей функцией для корреляторов. С точки зрения пространств модулей эта статистическая сумма является производящей функцией для топологических инвариантов многообра- 
зий $[22,21]$.

Существенно новые возможности для описания непертурбативной физики в калибровочных теориях в режиме сильной связи появились после получения Зайбергом и Виттеном точного эффективного действия в $N=2$ суперсимметричной теории Янга-Миллса (ЯМ) $[1,2]$. При получении эффективного действия потребовалось вычислить вклады от произвольного числа инстантонов, и решение оказалось первым примером в 4-мерных теориях, когда полная инстантонная сумма была найдена точно. Вторым сушественно новым результатом явилось точное вычисление масс стабильных состояний в данной теории при произвольном масштабе энергии. Напомним, что эта теория имеет ненулевые однопетлевые вклады и относится к классу асимптотически свободных теорий поля.

Несмотря на некоторую парадоксальность ситуации (явное суммирование по инстантонам не удалось выполнить до настоящего времени), уже не осталось сомнения в правильности полученного результата, выдерживающего различные проверки. При вычислении эффективного действия были использованы три принципа, позволившие однозначно получить окончательный результат: голоморфность [27, 28], дуальность [29], а также их совместность с ренормгрупповыми потоками. Из голоморфности следует, что полное действие имеет вид $\int \operatorname{Im} \mathcal{F}(\Psi)$ и зависит только от одной голоморфной функции $\mathcal{F}$, называемой препотенциалом. Принцип дуальности, который фиксирует связь между режимами сильной и слабой связи, естествен в конечной, например $N=4$, теории, когда очевидный модулярный параметр калибровочной теории, составленный из константы связи и $\theta$-члена,

$$
\tau=\frac{\mathrm{i}}{g^{2}}+2 \pi \theta
$$

(здесь и ниже i - мнимая единица), не перенормируется и совпадает со своим ультрафиолетовым значением. В теории с перенормировками формулировка дуальности в высшей степени нетривиальна. Прием, который на первый взгляд кажется несколько искусственным, состоит в следуюшем. В теорию вводится дополнительный объект - риманова поверхность высокого рода $[30,31]$, матрица периодов которой совпадает с матрицей констант связи в эффективной теории, зависяшей от значения параметра порядка в данном вакууме.

Наличие непрерывного семейства вакуумных состояний можно пояснить следуюшим образом. В $N=2$ теории затравочный лагранжиан для скалярных полей, преобразующихся по присоединенному представлению калибровочной группы, содержит потенциал

$$
V(\phi)=\operatorname{Tr}\left[\phi, \phi^{+}\right]^{2} .
$$

Так как суперсимметрия требует обрашения в нуль энергии основного состояния, то скалярные поля могут принимать любое значение в подалгебре Картана калибровочной группы $\phi=\operatorname{diag}\left(a_{1}, \ldots, a_{n_{c}}\right)$. Таким образом, мы вновь сталкиваемся с пространством коллективных координат - вакуумных средних скалярного поля, называемого кулоновской ветвью пространства модулей. Заметим, что естественно пользоваться калибровочно-инвариантными координатами $u_{k}=\operatorname{Tr}\left\langle\phi^{k}\right\rangle$. Выбор точки на кулоновской ветви эквивалентен выбору конкретного вакуума в теории поля и задает масштаб, на котором 
определена эффективная константа связи. В обшей точке пространства модулей после выпадения конденсата теория эффективно становится абелевой.

Риманова поверхность, описывающая теорию, вводится таким образом, чтобы координаты кулоновской ветви пространства модулей определяли точки ветвления и отображались в пространство модулей комплексных структур поверхностей. Матрица периодов поверхности связана указанным выше образом с константами связи, определенными именно в данной точке пространства модулей. Для введения новых объектов, обладаюших правильными трансформационными свойствами относительно преобразования дуальности, которое теперь преврашается в модулярное преобразование, необходимо определить на римановой поверхности подходящий дифференциал $d S$. Интегралы от данного дифференциала по циклам на поверхности

$$
a_{i}=\oint_{A_{i}} d S, \quad a_{D_{i}}=\oint_{B_{i}} d S, \quad \tau_{i j}(u)=\frac{\partial^{2} \mathcal{F}}{\partial a_{i} \partial a_{j}},
$$

где $i, j=1, \ldots, N_{c}-1$ для калибровочной группы $S U\left(N_{c}\right)$, преобразуются друг через друга при модулярных преобразованиях.

Было показано, что именно интегралы от дифференциала $d S$ по циклам определяют спектр стабильных состояний в теории, насыщающих границу Богомольного-ПрасадаСоммерфельда (БПС). Например, общая формула для БПС-спектра в $S U(2)$-теории имеет вид

$$
M_{n, m}^{2}=\left(n a\left(u_{2}\right)\right)^{2}+\left(m a_{D}\left(u_{2}\right)\right)^{2}
$$

где квантовые числа $n, m$ отвечают “электрическим" и “магнитным" состояниям. Не должно вызывать удивления то обстоятельство, что спектр, полученный из низкоэнергетического действия, фиксирует спектр масс сколь угодно тяжелых БПС-состояний. Дело в том, что БПС-спектр входит в центральньй заряд алгебры суперсимметрии [32] и, таким образом, имеет аномальное происхождение. С другой стороны, любые аномалии не перенормируются квантовыми поправками и могут быть вычислены как в ультрафиолетовой, так и в инфракрасной областях.

Оба вспомогательных объекта - риманова поверхность и дифференциал на ней - вводятся искусственно, поэтому попытка выяснить их роль в общем контексте описания вакуумного сектора суперсимметричных теорий и привела к обнаружению интегрируемых структур, ответственных за решение.

Прогресс в описании низкоэнергетических суперсимметричных калибровочных теорий инициировал изучение дуальности в теории струн. Оказалось, что для формулировки дуальности в теории струн необходимо ввести новые объекты, получившие название D-бран $[33,34]$. Открытые струны могут оканчиваться на D-бранах, поэтому на струны может быть наложено граничное условие Дирихле. Более того, D-бранам приписывается заряд в секторе рамон-рамоновских струн (RR-струн). C помощью D-бран струнная дуальность может быть определена самосогласованно, причем в теории струн может быть введено три типа дуальностей $[35,36]$.

Так как теория суперструн не содержит аномалий только в 10-мерном пространстве, возникает вопрос о роли оставшихся шести измерений. Результаты последних лет ясно 
указывают на то, что дополнительные размерности следует отождествлять с нулевыми модами скалярных полей [37], присутствующих в 4-мерных теориях поля. В наиболее обшей ситуации при $d=4$, отвечающей $N=4$ суперсимметричной теории поля, имеются три комплексных скалярных поля, нулевые гармоники которых следует воспринимать как координаты в 6-мерном пространстве. Вообще говоря, пространство полей имеет сложную структуру, и изучение допустимых метрик в этом пространстве является предметом многочисленных исследований.

С точки зрения теории поля наибольший интерес представляет ситуация, при которой гравитационные степени свободы в струне заморожены [38] и изучается только калибровочный сектор теории. Таким образом, для изучения обших 4-мерных калибровочных теорий необходимо ввести произвольную калибровочную группу, требуемую суперсимметрию и определить все параметры теории, например константы связи и массы. Оказывается, что учет бранных степеней свободы позволяет решить все задачи одновременно. Сушественными свойствами бранных состояний, которые позволяют получать подобный результат, являются наличие $U(1)$-калибровочного поля на мировой поверхности бран и существование их связанных состояний [37].

Опишем, каким образом 4-мерная теория поля может быть сфформулирована как теория на мировой поверхности браны некоторой размерности. В качестве исходной рассматривается 10-мерная теория суперструн с некоторой суперсимметрией, а браны являются БПС-состояниями, нарушаюшими суперсимметрию в два раза. Сушествуют два подхода к описанию теории поля. В первом подходе рассматривается теория в плоском пространстве и суперсимметрия нарушается фоновой бранной конфигурацией [39], во втором подходе, известном как "geometrical engineering" [40], суперсимметрия нарушается специальным выбором метрики дополнительного 6-мерного многообразия. Например, при использовании метрики многообразия К3 суперсимметрия нарушается вдвое.

Следуюшим шагом является получение калибровочной группы, например $S U(N)$, которая отсутствует в исходной теории струн. Вновь возможны два подхода: в первом из них группа возникает как следствие появления связанного состояния бран [37], во втором - как результат выбора компактного пространства со структурой сингулярностей, отвечаюшей системе корней некоторой группы [41]. Возникновение калибровочной группы высокого ранга при слиянии бран можно пояснить следующим образом. Каждая из бран несет $U(1)$-фактор, причем открытые струны могут быть натянуты между ними. Открытые струны содержат моды калибровочных полей, которые массивны, причем масса в силу механизма Хиггса пропорциональна расстоянию между бранами в объемлюшем пространстве. Если расстояние между бранами стремится к нулю, то ранг соответствующей калибровочной группы возрастает. В подходе "geometrical engineering" калибровочная группа возникает в результате наматывания браны на многообразие с соответствующей структурой сингулярностей.

Роль скалярных полей в теории поля играют координаты бран или связанного состояния бран в 6-мерном пространстве. Последним шагом при получении грубой картины теории поля является введение масс и констант связи. Массы вводятся в теорию до- 
бавлением бран другой коразмерности, причем роль масс играют координаты дополнительных бран в 6-мерном пространстве [39]. Аналогично в теорию вводятся и константы связи, более того, показывается, что их значение может быть отождествлено с расстоянием между фоновыми бранами в одной картине или размером компактных многообразий в подходе "geometrical engeneering" [40].

Наиболее прозрачным примером указанного подхода является реализация решения Зайберга-Виттена для $N=2$ суперсимметричной калибровочной теории в терминах бран. Теория в подходе IIA реализуется следующим образом [42]. Калибровочная теория с группой $S U(N)$ возникает на мировой поверхности $N$ параллельных D4-бран, вложенных в плоское 10-мерное пространство. Мировая поверхность бран 5-мерна, поэтому требуется описать оставшиеся пять измерений в терминах теории поля. Согласие с теорией поля достигается, если считать, что по трем координатам все браны локализованы в нуле, а голоморфная координата по двум измерениям, скажем $x_{4}+\mathrm{i} x_{5}$, является вакуумным значением комплексного скалярного поля в $N=2$ теории, т.е. координатой на кулоновской ветви пространства модулей.

Мы интересуемся 4-мерной теорией поля, поэтому D4-браны должны быть ограничены по одному измерению, например $x_{6}$. С этой целью в теорию вводятся две дополнительные 5-браны типа бран Невё-Шварца, на которых оканчиваются D4-браны. Именно такая конфигурация бран нарушает исходную суперсимметрию в 10-мерном пространстве до $N=2$ суперсимметрии в 4-мерном пространстве. Расстояние между фоновыми бранами вдоль координаты $x_{6}$ отождествляется с константой связи в калибровочной теории $\delta x_{6}=1 / g^{2}$, причем деформации фоновых бран могут быть отождествлены с пертурбативной перенормировкой заряда. В теорию может быть введена материя в фундаментальном представлении, для чего в бранную конфигурацию вводятся $N_{f}$ дополнительных D6-бран, координаты которых вдоль направления $x_{4}+\mathrm{i} x_{5}$ отождествляются с массами. Для введения материи в присоединенном представлении требуется, чтобы координата $x_{6}$ (точнее, ее комплексная версия) была периодической.

Описанная выше бранная конфигурация сингулярна, так как невозможно гладко описать пересечение D4-бран и фоновых 5-бран. Для разрешения этой проблемы Виттен предложил поднять конфигурацию в 11-мерное пространство, играющее для мембраны ту же роль, что и 10-мерное пространство для суперструны. Теория, определенная в 11-мерном пространстве, носит название М-теории, причем известно, что в низкоэнергетическом пределе она отвечает 11-мерной супергравитации $[43,44]$. Важным обстоятельством является то, что в М-теории имеются солитоны - М5-браны и мембраны. Дополнительное 11-е измерение предполагается компактным, и М5-брана, намотанная на дополнительное измерение, становится D4-браной в 10-мерном пространстве. Если М5-брана локализована по 11-му измерению, то она становится 5-браной в теории струн.

Приведенные аргументы приводят к следуюшей картине: все браны в теории типа IIA можно рассматривать как единую М5-брану в М-теории, намотанную на некоторую двумерную поверхность, вложенную в плоское пространство. Оказывается, что именно эта поверхность является римановой поверхностью, в терминах которой формулирует- 
ся решение Виттена-Зайберга. Таким образом, проясняется ее роль в описании вакуумной конфигурации поля. Более того, аналогичная картина в рамках М-теории была получена и для $N=1$ калибровочных теорий [45, 46], в которых конфайнмент реализуется через конденсацию монополей, что позволяет надеяться на адекватность бранной картины для несуперсимметричной КХД.

В другом подходе в рамках теории типа IIB [47], связанной с обсуждавшейся выше теорией IIA преобразованием $T$-дуальности [48], пространство, в которое вкладываются браны, обладает нетривиальной топологией, и браны предполагаются намотанными на некоторые циклы на поверхностях, например на эллиптические кривые, вложенные в многообразие К3 [49]. В этом подходе меньше наглядности, однако автоматически возникают топологические теории на кривых, которые генерируют многочастичные интегрируемые системы. Как и в картине IIA, бранная конфигурация становится гладкой, если учесть дополнительные размерности. При этом следует рассматривать 12-мерную теорию, носящую название F-теории [50]. Дополнительные две размерности предполагаются компактифицированными на тор, модулярньй параметр которого отождествляется с ультрафиолетовыми значениями константы связи и $\theta$-члена в калибровочной теории поля.

\section{2. МНОГОЧАСТИЧНЫЕ СИСТЕМЫ И КАЛИБРОВОЧНЫЕ ТЕОРИИ В ДВУХ И ТРЕХ ИЗМЕРЕНИЯХ}

В этом разделе показано, что многочастичные системы естественно появляются в двумерных и трехмерных калибровочных теориях, причем фазовое пространство систем частиц интерпретируется в терминах топологических степеней свободы калибровочных теорий. Ниже мы увидим, что двумерная теория ЯМ тесно связана с системой Калоджеро-Мозера [51-55], а трехмерная теория ЧС - с ее релятивистским аналогомсистемой Рюсенара [56, 57].

2.1. Многочастичные системы Калоджеро и теория ЯМ в двух измерениях. Покажем, каким образом лагранжева формулировка систем Калоджеро возникает из описания теории ЯМ на цилиндре $[58,59]$. Напомним, что система Калоджеро определена как система $N$ частиц с парным взаимодействием $[51,52,55]$ :

$$
V\left(x_{1}, \ldots, x_{N}\right)=\sum_{i \neq j} \frac{g^{2}}{\left(x_{i}-x_{j}\right)^{2}},
$$

$g^{2}$ - константа связи, удовлетворяюшая условию $g^{2}>-1 / 8$, обеспечиваюшему отсутствие падения на центр. Как уже упоминалось, фазовые пространства интегрируемых систем тесно связаны с групповыми многообразиями, поэтому в этом разделе мы будем изучать лагранжианы, имеющие групповой смысл.

Мы будем использовать здесь подход, основанньй на методе гамильтоновой редукции. Напомним основные элементы метода. На первом шаге мы выбираем симплектическое многообразие, на котором группа действует гамильтоновым образом. Действие группы сохраняет симплектическую структуру и определяет отображение момента 
из исходного фазового пространства в дуальную алгебру. Факторизация по действию группы исходного фазового пространства задает редуцированное фазовое пространство, причем свободная динамика на исходном фазовом пространстве отвечает нетривиальной эволюции на редуцированном пространстве.

Начнем рассмотрение с центрального расширения алгебры токов $\mathcal{L} \mathrm{g}$, где g - полупростая алгебра Ли. В качестве исходного фазового пространства мы выбираем кокасательное расслоение к алгебре, состоящее из данных $(A, \kappa ; \phi, c)$, где $\phi$ есть g-значное скалярное поле на окружности, $c$ - центральный элемент, $A$ - калибровочное поле на окружности, $\kappa$ - уровень, дуальный к $c$. Естественная скобка имеет вид

$$
\left[\left(\phi_{1}(\varphi), c_{1}\right),\left(\phi_{2}(\varphi), c_{2}\right)\right]=\left(\left[\phi_{1}(\varphi), \phi_{2}(\varphi)\right], \int_{S^{1}}\left\langle\phi_{1}, \partial_{\varphi} \phi_{2}\right\rangle\right)
$$

естественное спаривание между $\hat{\mathrm{g}}$ и $\hat{\mathrm{g}}^{*}$ определяется следующим образом:

$$
\langle(A, \kappa),(\phi, c)\rangle=\int_{S^{1}}\langle\phi, A\rangle+c \kappa,
$$

а симплектическая структура имеет вид

$$
\Omega=\int_{S^{1}} \operatorname{tr}(\delta \phi \wedge \delta A)+\delta c \wedge \delta \kappa
$$

Можно определить присоединенное и коприсоединенное действия групшы петель $\mathcal{L} G$ на $\hat{\mathrm{g}}$ и $\hat{\mathrm{g}}^{*}$ формулами

$$
\begin{aligned}
(\phi(\varphi), c) & \rightarrow\left(\mathrm{g}(\varphi) \phi(\varphi) \mathrm{g}(\varphi)^{-1}, \int_{S^{1}} \operatorname{tr}\left(-\phi \mathrm{g}^{-1} \partial_{\varphi} \mathrm{g}\right)+c\right) \\
(A, \kappa) & \rightarrow\left(\mathrm{g} A \mathrm{~g}^{-1}+\kappa \mathrm{g} \partial_{\varphi} \mathrm{g}^{-1}, \kappa\right)
\end{aligned}
$$

Действие группы сохраняет симплектическую структуру и, таким образом, задает структуру отображения момента

$$
\mu: T^{*} \hat{\mathrm{g}} \rightarrow \hat{\mathrm{g}}^{*}
$$

который отображает $(\phi, c ; A, \kappa)$ в пару $(\kappa d \phi+[A, \phi], 0)$.

Напомним, что фиксация уровня отображения момента имеет простой физический смысл: в теории обычного углового момента фиксируется сектор гильбертова пространства теории. В калибровочной теории - это выделение калибровочно-инвариантных состояний.

Рассмотрим элемент $J$ из $\mathrm{g}^{*}$, имеющий максимальный стабилизатор, отличный от $G$. Нетрудно убедиться, что этот элемент имеет вид

$$
J_{\nu}=\nu \sum_{\alpha \in \Delta_{+}}\left(e_{\alpha}+e_{-\alpha}\right) \text {, }
$$


где $\nu$ - некоторое число, $e_{\alpha}, e_{-\alpha}$ - элементы нильпотентной подалгебры $n_{ \pm} \subset \mathrm{g}$, соответствуюшей корням $\alpha$, а $\Delta_{+}-$система положительных корней. Обозначим орбиту элемента $J_{\nu}$ через $\mathcal{O}_{\nu}$, а через $R_{\nu}$ - представление $G$, возникаюшее при квантовании $\mathcal{O}_{\nu}$. Для общих орбит $J \in \mathrm{g}^{*}$ обозначим через $G_{J}$ стабилизатор $J$ орбиты $\mathcal{O}_{J}$, т.е. $\mathcal{O}_{J}=G / G_{J}$. Таким образом, подходящая орбита в аффинном случае $\mathcal{O}_{\nu}$,

$$
\mu=(\mathcal{J}[\mu], 0): \mathcal{J}[\mu](\varphi)=\delta(\varphi) J_{\nu}
$$

конечномерна.

Редукция требует разрешения отображения момента, который в афффинном случае имеет смысл закона Гаусса

$$
\kappa \partial_{\varphi} \phi+[A, \phi]=J_{\mu}(\varphi)=\delta(\varphi) J_{\nu}
$$

Связь удобно разрешать следуюшим образом. Сначала с помошью обшего калибровочного преобразования $\tilde{\mathrm{g}}(\varphi)$ калибровочное поле $A$ можно привести к подалгебре Картана $D$. Оставшаяся калибровочная свобода связана с постоянными калибровочными преобразованиями, не меняюшими $D$. Заметим, что выбор $D$ не единствен и параметризуется инвариантом действия $\mathcal{L} G$ - классами сопряженности монодромии $\exp ((2 \pi / \kappa) D) \in \mathbf{T} \subset G$. Зафиксируем класс сопряженности, обозначив через і $x_{i}$ элементы матрицы $D=\mathrm{i} X$. Удобно разложить g-значную функцию $\phi$ на $S^{1}$ на картановскую $P(\varphi) \in t$ и нильпотентную $\phi_{ \pm}(\varphi) \in n_{ \pm}$части. Пусть $\phi_{\alpha}=\left\langle\phi, e_{\alpha}\right\rangle$, тогда в этих терминах соотношение (2.7) принимает вид

$$
\begin{aligned}
\kappa \partial_{\varphi} P & =\delta(\varphi)\left[J_{\nu}^{\mathrm{g}}\right]_{\gamma}, \\
\kappa \partial_{\varphi} \phi_{\alpha}+\left\langle D, \phi_{\alpha}\right\rangle & =\delta(\varphi)\left[J_{\nu}^{\mathrm{g}}\right]_{\alpha},
\end{aligned}
$$

где $J_{\nu}^{\tilde{\mathrm{g}}} \in \operatorname{Ad}_{\tilde{\mathrm{g}}(0)}^{*}\left(J_{\nu}\right),[J]_{\gamma}$ обозначает картановскую часть $J$, а $[J]_{\alpha}=\left\langle J, e_{\alpha}\right\rangle$.

Из уравнения (2.8) получаем $D=$ const и $\left[J_{\nu}^{g}\right]_{\gamma}=0$. Таким образом, мы можем привести $J_{\nu}^{\mathrm{g}}$ к $J_{\nu}$. Согласно $(2.9)$ при $\varphi \neq 0$ функцию $\phi_{\alpha}(\varphi)$ можно представить в следующем виде:

$$
\phi_{\alpha}(\varphi)=\exp \left(-\frac{\varphi}{\kappa}\langle D, \alpha\rangle\right) \times M_{\alpha}
$$

где $M_{\alpha}$ - локально-постоянный вектор в g. Очевидно, что $M_{\alpha}$ испытьвает скачок при $\varphi=0$, равньй

$$
\left[\exp \left(-\frac{2 \pi}{\kappa}\langle D, \alpha\rangle\right)-1\right] \times M_{\alpha}=\left[J_{\nu}^{\mathrm{g}}\right]_{\alpha} .
$$

Окончательно приходим к следуюшему результату: физическими степенями свободы являются компоненты матриц $\exp (-(2 \pi \mathrm{i} / \kappa) X)$ и $P$ с симплектической структурой

$$
\omega=\frac{1}{2 \pi \mathrm{i}} \operatorname{tr}(\delta P \wedge \delta X)
$$

причем

$$
\phi_{\alpha}(\varphi)=\nu \frac{\exp \left(-\frac{\mathrm{i} \varphi}{\kappa}\langle X, \alpha\rangle\right)}{\exp \left(-\frac{2 \pi \mathbf{i}}{\kappa}\langle X, \alpha\rangle\right)-1}
$$


Теперь можно построить простую гамильтонову систему на $T^{*} \hat{\mathrm{g}}$ с гамильтонианом, инвариантным относительно действия $(2.3),(2.4)$, которая индуцирует относительно сложную систему на редуцированном симплектическом многообразии $T^{*} \mathbf{T}$. Собрав вместе член, задающий пуассонову структуру, отображение момента (закон Гаусса) с множителем Лагранжа $A_{0}$ и квадратичный оператор Казимира

$$
\mathcal{H}_{2}=\frac{1}{4 \pi} \int_{S^{1}} d \varphi\langle\phi, \phi\rangle
$$

мы немедленно узнаем двумерную теорию ЯМ в гамильтоновой формулировке с дополнительной вильсоновской линией

$$
L=\int_{S^{1}} d \varphi d t\left(\operatorname{Tr} A \partial_{t} \phi+\langle\phi, \phi\rangle+\operatorname{Tr} A_{0}\left(\kappa \partial_{\varphi} \phi+[A, \phi]-\delta(\varphi) J_{\nu}\right)\right) .
$$

На редуцированном многообразии мы получаем тригонометрическую систему Калоджеро-Сазерленда [55] с гамильтонианом

$$
H_{2}=-\frac{1}{2} \operatorname{tr} P^{2}+\sum_{\alpha \in \Delta_{+}} \frac{\nu^{2}}{\sin ^{2}\langle X, \alpha\rangle},
$$

где $\Delta_{+}$обозначает систему положительных корней группы g. Например, для $G=$ $S U(N)$ получаем гамильтониан с парным взаимодействием

$$
V_{i j}^{A}=\frac{g^{2}}{\sin ^{2}\left(x_{i}-x_{j}\right)},
$$

а для $G=S O(2 N)$ имеем

$$
V_{i j}^{D}=g_{2}^{2}\left[\frac{1}{\sin ^{2}\left(x_{i}-x_{j}\right)}+\frac{1}{\sin ^{2}\left(x_{i}+x_{j}\right)}\right]+g_{1}^{2}\left[\frac{1}{\sin ^{2} x_{i}}+\frac{1}{\sin ^{2} x_{j}}\right],
$$

где $g_{1}, g_{2}$ - константы связи. В общем случае число независимых констант связи совпадает с числом независимых орбит группы Вейля.

2.2. Системы Рюсенара и калиброванная $G / G \sigma$-модель. Покажем, что системы Калоджеро допускают естественную релятивизацию, которая также имеет описание на языке теории поля. Соответствуюшей теорией поля является калиброванная G/G $\sigma$-модель, которая, в свою очередь, эквивалентна лагранжиану ЧС [60]. Параметром деформации с точки зрения групш петель является уровень алгебры Каца-Муди.

Заметим, что релятивизация возникает в ряде физических задач, например в рассеянии солитонов в двумерных системах [61] и в калибровочных теориях в старших измерениях [62, 63], поэтому единое лагранжево описание разных физических ситуаций несомненно полезно для приложений.

Как и в нерелятивистском случае, мы будем работать в рамках метода гамильтоновой редукции, но теперь в качестве нередуцированного фазового пространства выбирается кокасательное расслоение к центрально-расширенной группе петель $\widehat{G}$. Как 
симплектическое многообразие его можно представить данными $\left(\mathrm{g}: S^{1} \rightarrow G, c \in U(1)\right.$ : $\left.A \in \Omega^{1}\left(S^{1}\right) \otimes \mathrm{g}^{*}, \kappa \in \mathbb{R}\right)$. Действие на нем группы определено следуюшим образом:

$$
\mathrm{g} \rightarrow h \mathrm{~g} h^{-1}, \quad A \rightarrow h A h^{-1}+\kappa h \partial h^{-1}, \quad \kappa \rightarrow \kappa, \quad c \rightarrow c \times \mathcal{S}(\mathrm{g}, h),
$$

где $\mathcal{S}$ строится по коциклу на группе $\mathcal{L} G, \mathcal{S}(\mathrm{g}, h)=\Gamma(h, \mathrm{~g}) \Gamma\left(h \mathrm{~g}, h^{-1}\right)$.

Симплектическая структура, инвариантная относительно действия группы, имеет вид

$$
\begin{aligned}
\Omega= & \int_{S^{1}} \operatorname{tr}\left[A\left(\mathrm{~g}^{-1} \delta \mathrm{g}\right)^{2}+\delta A \wedge \mathrm{g}^{-1} \delta \mathrm{g}\right]+ \\
& +\int_{S^{1}} \operatorname{tr}\left[\kappa \partial_{\varphi} \mathrm{g} \dot{\mathrm{g}}^{-1}\left(\delta \mathrm{gg}^{-1}\right)^{2}-\kappa \delta\left(\partial_{\varphi} \mathrm{g}\right) \dot{\mathrm{g}}^{-1} \delta \mathrm{gg}^{-1}\right]+c^{-1} \delta c \wedge \delta \kappa
\end{aligned}
$$

Обобшение закона Гаусса может быть найдено из действия группы:

$$
\mu(\mathrm{g}, c ; A, \kappa)=\left(\mathrm{g} A \mathrm{~g}^{-1}+\kappa \mathrm{g} \partial \mathrm{g}^{-1}-A, 0\right)
$$

Следуюший шаг состоит в фиксации уровня отображения момента. Естественно предположить, что его цветовая структура не меняется, сохраняя интегрируемость:

$$
\mu(\mathrm{g}, c ; A, \kappa)=\mathrm{i} \nu\left(\frac{1}{N} I d-e \otimes e^{+}\right) \delta(\varphi)
$$

Как и раньше, обшим калибровочным преобразованием $H(\varphi)$ мы можем привести матрицу $A$ к диагональному виду $D$ по модулю действия аффиннной группы Вейля.

Отображение момента принимает вид

$$
\mathrm{g} D \mathrm{~g}^{-1}+\kappa \mathrm{g} d \mathrm{~g}^{-1}-D=\mathrm{i} \nu\left(\frac{1}{N} I d-f \otimes f^{+}\right) \delta(\varphi)
$$

где $f=H e$ - вектор в $\mathbb{C}^{N}$ с единичной нормой $\langle f, f\rangle=1$. Остаточная калибровочная симметрия позволяет получить $f \in \mathbb{R}^{N}$ и $A$, после чего из (2.19) немедленно следует

$$
\mathrm{g}=\exp \left(\frac{\varphi}{\kappa} D\right) G(\varphi) \exp \left(-\frac{\varphi}{\kappa} D\right), \quad \partial_{\varphi} G=-\frac{J}{\kappa} G \delta(\varphi)
$$

где

$$
J=\mathrm{i} \nu\left(\frac{1}{N} I d-f \otimes f^{+}\right) .
$$

Удобно ввести монодромию связности $D$ :

$$
Z=\exp \left(-\frac{2 \pi}{\kappa} D\right)=\operatorname{diag}\left(z_{1}, \ldots, z_{N}\right), \quad \prod_{i} z_{i}=1, \quad z_{i}=\exp \left(\frac{2 \pi \mathrm{i} q_{i}}{\kappa}\right)
$$

с граничным условием

$$
\widetilde{G}^{-1} Z \widetilde{G}=\exp \left(\frac{2 \pi J}{\kappa}\right) Z
$$



где $\lambda=\exp (2 \pi \mathrm{i} \nu / N \kappa), \widetilde{G}=G(+0)$.

Решение уравнения удобно записать в терминах характеристического полинома матрицы $Z$

$$
P(z)=\prod_{i}\left(z-z_{i}\right)
$$

Пусть

$$
Q^{ \pm}(z)=\frac{P\left(\lambda^{ \pm 1} z\right)-P(z)}{\left(\lambda^{ \pm N}-1\right) z P^{\prime}(z)}
$$

тогда при $\lambda \rightarrow 1$ рациональные функции $Q^{ \pm}(z)$ стремятся к $1 / N$. В этих обозначениях матрица $\widetilde{G}$ переписывается в виде

$$
\begin{aligned}
\widetilde{G}_{i j}= & -\lambda^{-\frac{N-1}{2}} \frac{\lambda^{-N}-1}{\lambda^{-1} z_{i}-z_{j}} e^{\mathrm{i} \theta_{i}}\left(Q^{+}\left(z_{i}\right) Q^{-}\left(z_{j}\right)\right)^{\frac{1}{2}}= \\
= & \exp \left[\mathrm{i} \theta_{i}-\frac{\pi \mathrm{i}}{\kappa}\left(q_{i}+q_{j}\right)\right] \frac{\sin (\pi \nu / \kappa)}{\sin \left(\left(\pi q_{i j}-\nu / N\right) / \kappa\right)} \times \\
& \times \prod_{k \neq i, l \neq j} \frac{\sin \left(\left(\pi q_{i k}+\nu / N\right) / \kappa\right)}{\sin \left(\pi q_{i k} / \kappa\right)} \frac{\sin \left(\left(\pi q_{i l}-\nu / N\right) / \kappa\right)}{\sin \left(\pi q_{i l} / \kappa\right)},
\end{aligned}
$$

где переменные $\theta_{i}$ становятся импульсами частиц с координатами $q_{i}$. Таким образом, симплектическая структура на редуцированном многообразии пропорциональна $\sum_{i} d \theta_{i} \wedge d q_{i}$.

В качестве гамильтониана динамической системы на $T^{*} \widehat{G}$, инвариантного относительно действия группы, можно выбрать

$$
H_{\chi}=\int_{S^{1}} d \varphi \chi(\mathrm{g})
$$

Например, функция $\chi_{ \pm}(\mathrm{g})=\operatorname{Tr}\left(\mathrm{g} \pm \mathrm{g}^{-1}\right)$ (след берется по фундаментальному представлению $S U(N))$ дает

$$
H_{ \pm}=\sum_{i}\left(e^{\mathrm{i} \theta_{i}} \pm e^{-\mathrm{i} \theta_{i}}\right) \prod_{j \neq i} f\left(q_{i j}\right),
$$

где функция $f(q)$ определяется следуюшим образом:

$$
f^{2}(q)=\left[1-\frac{\sin ^{2}(\pi \nu / \kappa N)}{\sin ^{2}(\pi q / \kappa)}\right]
$$

что приводит к модели Рюсенара, а в пределе $\kappa \rightarrow \infty$ мы возврашаемся к модели Калоджеро-Сазерленда.

Теперь можно ответить на вопрос, какой теории поля отвечает наша многочастичная система. В качестве действия выбираем стандартный член $\int p d q$, к которому добавлено отображение момента с множителем Лагранжа:

$$
\begin{aligned}
S(A, \mathrm{~g})= & \int d \varphi d t \operatorname{tr}\left[-A_{\varphi} \mathrm{g}^{-1} \partial_{t} \mathrm{~g}-\kappa \partial_{t} \mathrm{~g} \cdot \mathrm{g}^{-1} \cdot \partial_{\varphi} \mathrm{g} \cdot \mathrm{g}^{-1}+\right. \\
& \left.+\kappa d^{-1}\left(\partial_{\varphi} \mathrm{g} \cdot \mathrm{g}^{-1}\left(d \mathrm{~g} \cdot \mathrm{g}^{-1}\right)^{2}\right)+A_{t}\left(\kappa \mathrm{g}^{-1} \partial_{\varphi} \mathrm{g}+\mathrm{g}^{-1} A_{\varphi} \mathrm{g}-A_{\varphi}\right)\right] .
\end{aligned}
$$


Нетрудно узнать в нем действие $G / G$ калиброванной $\sigma$-модели Весса-Зумино. Теория снова содержит вильсоновскую линию в представлении $R_{\nu}$ и предложенный выше гамильтониан (2.22). Для вычисления функционального интеграла на цилиндре, как и в случае теории ЯМ, удобно разрезать цилиндр по вильсоновской линии, после чего в силу инвариантности относительно группы выражение для ядра эволюции принимает вид суммы по характерам, причем сумма берется только по интегрируемым представлениям $S U(N)_{k}$.

Полезно рассмотреть эквивалентное представление в терминах теории ЧС с некоторым гамильтонианом. Теория ЧС должна быть определена на трехмерном многообразии, являюшемся произведением интервала на тор: $X=I \times T^{2}$, с действием

$$
S_{\mathrm{CS}}=\frac{\mathrm{i} \kappa}{4 \pi} \int_{X} \operatorname{tr}\left(A \wedge d A+\frac{2}{3} A \wedge A \wedge A\right) .
$$

Фазовым пространством такой теории является пространство модулей плоских связностей на торе с отмеченной точкой соответствующей вильсоновской линии, которая модифицирует функциональный интеграл:

$$
\mathcal{D} A\left\langle v_{1}\left|T_{R}\left(P \exp \int A\right)\right| v_{2}\right\rangle \exp \left(-S_{\mathrm{CS}}(A)\right) .
$$

Монодромия вокруг отмеченной точки $U$ задается старшим весом $\hat{h}$ представления $R_{\nu}$ следуюшим образом:

$$
U=\exp \left(\frac{2 \pi \mathrm{i}}{\kappa+N} \operatorname{diag}\left(\hat{h}_{i}\right)\right) .
$$

Условие, накладываемое на монодромии на торе $\Sigma=T^{2}$, имеет вид

$$
\mathrm{g}_{A} \mathrm{~g}_{B} \mathrm{~g}_{A}^{-1} \mathrm{~g}_{B}^{-1}=\mathrm{g}_{C},
$$

где $\mathrm{g}_{A}, \mathrm{~g}_{B}$ - монодромии по циклам, a $\mathrm{g}_{C}$ - монодромия вокруг отмеченной точки.

Важным обстоятельством является эквивалентность наблюдаемых, зависящих от $A_{t}$ (где $\partial_{t}$ касательно $S^{1}$ ), в теории ЧС на $\Sigma \times S^{1}$ и в $G / G$ теории Весса-Зумино-Виттена. С помощью этой эквивалентности получим спектр модели Рюсенара, который совпадает со спектром релятивистских частищ на окружности. В чистой теории ЧС частицы имеют фермионную статистику в соответствии с фазовым пространством $\left(\mathrm{T}^{N-1} \times\right.$ $\left.\mathbf{T}^{N-1}\right) / \mathcal{S}_{N}$, где $\mathbf{T}^{N-1}$ - тор Картана в $S U(N)$, a $\mathcal{S}_{N}$ - симметрическая группа. Очевидно, что уравнение Шредингера является разностным, так как $\cos \left(2 \pi \mathrm{i} \partial_{q} /(\kappa+N)\right)-$ конечно-разностный оператор. Собственными значениями этого оператора на окружности являются матрицы $\exp (2 \pi i n q)$ с собственными значениями

$$
E_{n}=\cos \left(\frac{2 \pi n}{\kappa+N}\right) .
$$

Поэтому полный спектр системы имеет вид $\sum_{i} E_{n_{i}}$, где мы учли условия, следующие из инвариантности $E_{n}=E_{n+\kappa+N}$ и симметрии:

$$
\begin{gathered}
(\kappa+N)>n_{N}>\cdots>n_{i}>\cdots>n_{1} \geqslant 0, \\
\sum_{i} n_{i}<\kappa+N .
\end{gathered}
$$


2.3. Эллиптическая модель Калоджеро и калибровочные теории. Существует еше одно обобшение тригонометрической модели Калоджеро - эллиптическая модель, в которой решения уравнений движения формулируются в терминах римановых поверхностей - поверхностей уровня всех интегралов движения. В разделе 3 мы столкнемся с ситуацией, когда именно эллиптические системы оказываются ответственными за непертурбативную динамику в вакуумном секторе в суперсимметричных теориях ЯМ в четырех измерениях.

Здесь мы покажем, каким образом эллиптические модели описываются в терминах трехмерных голоморфных теорий в подходе гамильтоновой редукции [64]. В качестве затравочного фазового пространства выбирается кокасательное расслоение к алгебре $S L(N, \mathbb{C})$-значных функций на эллиптической кривой с модулярным параметром $\tau$. Фазовое пространство $T^{*} \hat{\mathrm{g}}^{\Sigma_{\tau}}$ представляется в виде

$$
(\phi, c ; \kappa \bar{\partial}+\bar{A}), \quad \phi: \Sigma_{\tau} \rightarrow s l_{N}(\mathbb{C}), \quad \bar{A} \in \Omega^{(0,1)}\left(\Sigma_{\tau}\right) \otimes s l_{N}(\mathbb{C}), \quad c, \kappa \in \mathbb{C},
$$

где $\omega$ - голоморфный дифференциал с интегралами по $A$ - и $B$-циклам, равными 1 и $\tau$, соответственно.

На исходном фазовом пространстве действует группа токов $S L_{N}(\mathbb{C})^{\Sigma_{\tau}}$ :

$$
\begin{aligned}
\phi(z, \bar{z}) & \rightarrow \mathrm{g}(z, \bar{z}) \phi(z, \bar{z}) \mathrm{g}(z, \bar{z})^{-1} \\
\bar{A}(z, \bar{z}) d \bar{z} & \rightarrow \mathrm{g}(z, \bar{z}) \bar{A}(z, \bar{z}) \mathrm{g}(z, \bar{z})^{-1}+\kappa \mathrm{g}(z, \bar{z}) \bar{\partial} \mathrm{g}(z, \bar{z})^{-1} \\
\kappa & \rightarrow \kappa, \quad c \rightarrow c+\int_{\Sigma_{\tau}} \omega \wedge \operatorname{tr}(\phi \mathrm{g} \bar{\partial} \mathrm{g}),
\end{aligned}
$$

сохраняюшая на $T^{*} \hat{\mathrm{g}}^{\Sigma_{\tau}}$ голоморфную симплектическую форму

$$
\Omega=\delta c \wedge \delta \kappa+\int_{\Sigma_{\tau}} \omega \operatorname{tr}(\delta \phi \wedge \delta \bar{A})
$$

и задаюшая отображение момента

$$
\mu=\kappa \bar{\partial} \phi+[\bar{A}, \phi]
$$

При описании системы взаимодействующих частиц мы опять расширяем фазовое пространство конечномерной коприсоединенной орбитой $\mathcal{O}_{\nu}^{-}$. Отображение момента $M$ на расширенном многообразии $X_{\nu}$ имеет вид

$$
0=\kappa \bar{\partial} \phi+[\bar{A}, \phi]-\nu\left(\mathbf{1}-u \otimes v^{*}\right) \frac{\delta(z, \bar{z}) d z \wedge d \bar{z}}{\omega}
$$

После калибровочных преобразований уравнение на матричные элементы имеет вид

$$
\kappa \bar{\partial} \phi_{i j}+a_{i j} \phi_{i j}=\nu\left(\delta_{i j}-u_{i} v_{j}^{*}\right) \delta(z, \bar{z})
$$

где $a_{i j}=a_{i}-a_{j}, a_{i} \in \mathbb{C}$ - диагональные элементы $\bar{A}$. Из уравнения $(2.29)$ можно получить следуюшие свойства матрицы $\phi$ : 
a) $1-u_{i} v_{i}^{*}=0$;

б) $\phi_{i i}=p_{i}=$ const, $i=1, \ldots, N, \sum_{i} p_{i}=0$;

в) для $i \neq j$

$$
\phi_{i j}=-\frac{2 \pi \mathrm{i} \nu}{\kappa} \frac{u_{i}}{u_{j}} \exp \left(\frac{a_{i j}(z-\bar{z})}{\kappa}\right) \frac{\theta_{11}\left(z+\pi a_{i j} / \kappa \tau_{2}\right)}{\theta_{11}(z) \theta_{11}\left(\pi a_{i j} / \kappa \tau_{2}\right)},
$$

где $\tau_{2}=\operatorname{Im} \tau, \theta_{11}$ - тэта-функция, обращающаяся в нуль в точке 0 .

Для доказательства свойства "в" сделаем подстановку

$$
\phi_{i j}(z, \bar{z})=\exp \left(\frac{a_{i j}(z-\bar{z})}{\kappa}\right) \psi_{i j}(z, \bar{z}),
$$

где $\psi_{i j}$ имеет нетривиальную монодромию

$$
\begin{aligned}
\psi_{i j}(z+1, \bar{z}+1) & =\psi_{i j}(z, \bar{z}) \\
\psi_{i j}(z+\tau, \bar{z}+\bar{\tau}) & =\exp \left(-\frac{2 \tau_{2} \mathrm{i}}{\kappa} a_{i j}\right) \psi_{i j}(z, \bar{z})
\end{aligned}
$$

Согласно (2.29) мы имеем $\bar{\partial} \psi_{i j}=-\left(\nu u_{i} / u_{j}\right) \delta(z, \bar{z})$, т.е. $\psi_{i j}$-мероморфное сечение голоморфного линейного расслоения с полюсом в нуле. Решение оказывается единственным и дается формулой (2.30). После выполнения процедуры редукции значение $\psi$, вычисленное для $z \neq 0$, оказывается матрицей Лакса, найденной ранее Кричевером.

Инварианты матрицы $\phi(z, \bar{z})$ задают гамильтонианы интегрируемой модели $\mathcal{M}$, первый из которых является гамильтонианом эллиптической модели Калоджеро-Мозера

$$
\frac{1}{2} \operatorname{tr} \phi(z, \bar{z})^{2}=\sum_{i} \frac{1}{2} p_{i}^{2}+\frac{4 \pi^{2} \nu^{2}}{\kappa^{2}}\left[\sum_{i<j} \wp\left(\frac{\pi a_{i j}}{\tau_{2} \kappa}\right)-\frac{N(N-1)}{2} \wp(z)\right] .
$$

Отметим, что описание эллиптической системы Рюсенара в подходе гамильтоновой редукции было получено в работе [65].

Опишем теперь эллиптическую деформацию двумерной теории ЯМ. Соответствующее действие имеет вид

$$
S_{\tau}=\int_{\Sigma_{\tau} \times S} \omega \wedge \operatorname{tr}\left(\phi F_{t \bar{z}}-\varepsilon \phi^{2}\right)
$$

где $F_{t \bar{z}}=\partial_{t} \bar{A}-\bar{\partial} A_{t}+\left[A_{t}, \bar{A}\right]-$ компонента напряженности калибровочного поля, $\omega=$ $d z$ - голоморфный дифференциал на $\Sigma_{\tau}$. Вертикальная вильсоновская линия $P \exp \int A_{t}$ в представлении $R_{\nu}$ приводит к искомой формулировке модели. Заметим, что эллиптическая модель Калоджеро является естественным обобшением систем Хитчина на пространстве модулей голоморфных векторных расслоений на поверхностях с отмеченными точками $[66,67]$. Другим известным пределом интегрируемых систем частищ являются системы типа цепочек Тоды. Оказывается, что периодические системы Тоды могут быть получены вырождением из эллиптической модели Калоджеро [68]. Для этого необходимо ввести новые переменные $a_{i}=x_{i}+(j-1) b / \kappa$, переопределить константу связи $\nu=\nu_{0} e^{b / \kappa}$ и перейти к пределу $b \rightarrow \infty$. В дальнейшем мы увидим, что этот предел соответствует явлению размерной трансмутации в 4-мерной теории поля. 
ИНТЕГРИРУЕМЫЕ МНОГОЧАСТИЧНЫЕ СИСТЕМЫ И КАЛИБРОВОЧНЫЕ ТЕОРИИ 21

\section{3. ДУАЛЬНОСТИ В МНОГОЧАСТИЧНЫХ СИСТЕМАХ И КАЛИБРОВОЧНЫЕ ТЕОРИИ}

Дуальность интенсивно изучается в контексте суперсимметричных калибровочных теорий, где она реализуется как инволюция, отображающая наблюдаемые одной теории в наблюдаемые другой теории. Дуальность оказьвается эффективной в случае, если константы связи теорий взаимно обратны, т.е. когда режиму слабой связи отвечает режим сильной связи. Зная связь многочастичных систем с калибровочными теориями, естественно получить полную группу дуальностей в интегрируемой системе. Установление подобной связи оказалось полезным и для калибровочных теорий, и для динамических систем [69, 70].

3.1. $T$-дуальность и разделение переменных. Имеются три сушественно различных типа дуальностей, проявляюшихся в системах Хитчина. Начнем с изучения аналога $T$-дуальности, которая может быть отождествлена с явлением разделения переменных в динамических системах [69]. Разделение переменных в интегрируемой системе позволяет свести систему с $N$ степенями свободы к $N$ идентичным системам с одной степенью свободы.

Недавно Склянин [71] сформулировал метод разделения переменных в системах, имеюших представление Лакса, который сводится к изучению динамики полюсов функции Бейкера-Ахиезера $\Psi(z, \lambda)$,

$$
L(z) \Psi(z, \lambda)=\lambda(z) \Psi(z, \lambda),
$$

при специальной нормировке. Полюсы $z_{i}$ функции $\Psi(z, \lambda)$ и значения $\lambda_{i}=\lambda\left(z_{i}\right)$ оказываются разделенными переменными.

Напомним, что фазовое пространство системы Хитчина отождествляется с кокасательным расслоением к пространству модулей голоморфных векторных расслоений $T^{*} M$ на поверхности $\Sigma$. Явление разделения переменных связано с существованием следуюших симплектоморфизмов. Фазовое пространство допускает еше два описания: как пара $(C, \mathcal{L})$, где $C$ - спектральная кривая динамической системы с линейным расслоением $\mathcal{L}$, или как гильбертова схема точек на $T^{*} \Sigma$, где число точек определяется рангом калибровочной группы. Именно последнее описание отвечает динамике в разделенных переменных. Описание гильбертовых схем на $T^{*} \Sigma$ в контексте систем Хитчина было получено для поверхностей без отмеченных точек в работе [72] и применено для систем типа Калоджеро $[69,73,74]$. В общем случае явление разделения переменных может быть сформулировано как симплектоморфизм между пространствами модулей расслоений, имеющих разные классы Черна.

В качестве примера рассмотрим пространство модулей $\mathcal{M}_{\vec{v}}$ стабильных когерентных пучков без кручения $\mathcal{E}$ на $S$. Пусть $\hat{A}_{S}=1-[\mathrm{pt}] \in H^{*}(S, \mathbb{Z})$ отождествляется с $A$-родом $S$. Вектор $\vec{v}=C h(\mathcal{E}) \sqrt{\hat{A}_{S}}=(r ; \vec{w} ; d-r) \in H^{*}(S, \mathbb{Z}), \vec{w} \in \Gamma^{3,19}$, отвечает пучкам с классами Черна:

$$
\begin{aligned}
& c h_{0}(\mathcal{E})=r \in H^{0}(S ; \mathbb{Z}), \\
& \operatorname{ch}_{1}(\mathcal{E})=\vec{w} \in H^{2}(S ; \mathbb{Z}), \\
& \operatorname{ch}_{2}(\mathcal{E})=d \in H^{4}(S ; \mathbb{Z}) .
\end{aligned}
$$


Теория струн, компактифицированная на $S$, обладает пространством модулей вакуумов

$$
\mathcal{M}_{A}=O\left(\Gamma^{4,20}\right) \backslash O(4,20 ; \mathbb{R}) / O(4 ; \mathbb{R}) \times O(20 ; \mathbb{R})
$$

с арифметической группой $O\left(\Gamma^{4,20}\right)$, которая отображает состояния, отвечающие разным векторам $\vec{v}$, друг в друга. Единственным инвариантом действия группы является величина $\vec{v}^{2}$.

Можно рассмотреть три реализации интегрируемой системы. В первой реализации мы изучаем пару $(\mathcal{E}, \phi)$, где $\mathcal{E}$ - векторное расслоение ранга $r$ над $\Sigma$ степени $l$, а $\phi-$ голоморфное сечение $\omega_{\Sigma}^{1} \otimes \operatorname{End}(\mathcal{E})$. Вторая версия включает пару $(C, \mathcal{L})$, где $C$ - кривая в $S$, реализующая класс гомологий $r[\Sigma]$, а $\mathcal{L}$ - линейное расслоение на $C$. В третьей реализации мы имеем гильбертову схему точек на $S$ длины $h$, где $h=(\operatorname{dim} \mathcal{M}) / 2$. Эквивалентность первой и второй реализаций следует из факта, что связанное состояние $N$ штук D2-бран, намотанных на $\Sigma$, представляется одной D2-браной, намотанной на голоморфную кривую, $N$-кратно накрывающую $\Sigma$. Эквивалентность второго и третьего описаний естественным образом интерпретируется как преобразование $T$-дуальности.

Заметим, что разделение переменных проливает дополнительный свет на дуальность Ленглэндса, формулируемую в терминах спектра систем Хитчина. Дуальность Ленглэндса как квантовое разделение переменных была сформулирована в [75]. Рассмотрение, проведенное в работе [70], позволяет сформулировать гипотезу о том, что правильньй классический аналог этой дуальности - это симплектоморфизм, сводящий фазовое пространство системы к гильбертовой схеме точек на 4-мерном многообразии.

3.2. $S$-дуальность. Покажем, что $S$-дуальность, сформулированная в теории поля, имеет ясную интерпретацию для голоморфной интегрируемой системы. Переменные действия в динамической системе описываются интегралами от мероморфного дифференциала $\lambda$ по $A$-циклам на спектральной кривой. C другой стороны, в качестве независимых периодов можно выбрать интегралы от $\lambda$ по любому лагранжеву подпространству в $H_{1}\left(\mathbf{T}_{b} ; \mathbb{Z}\right)$. Таким образом, мы получаем следуюшую структуру переменных действия. Локально над диском выбираются базис в $H_{1}$ в слое и система $A$-циклов. Выбор может зависеть от диска, поэтому на пересечении дисков возникают преобразования $S p(2 m, \mathbb{Z})$, связывающие базы, задаюшие $S p(2 m, \mathbb{Z})$-расслоение. Исходя из свойств матрицы периодов, нетрудно показать, что форма

$$
d I^{i} \wedge d I_{i}^{D}
$$

обрашается в нуль. Таким образом, локально можно найти функцию, называемую препотенциалом $\mathcal{F}$, такую, что

$$
I_{i}^{D}=\frac{\partial \mathcal{F}}{\partial I^{i}}
$$

Для иллюстрации $A A$-дуальности рассмотрим двухчастичную систему, ответственную за $\mathcal{N}=2 S U(2)$-суперсимметричную калибровочную теорию:

$$
H=\frac{p^{2}}{2}+\Lambda^{2} \cos q,
$$


где коэффициент $\Lambda^{2}$ играет роль константы связи в двухчастичной задаче и одновременно задает масштаб в теории поля. Переменные действия определяются интегралами от дифференциала $p d q$. Введем обозначения $x=\cos q, y=p \sin q /(\sqrt{-2} \Lambda), u=H / \Lambda^{2}$, в терминах которых спектральная кривая имеет вид

$$
y^{2}=(x-u)\left(x^{2}-1\right)
$$

и совпадает с кривой Виттена-Зайберга с периодами

$$
I=\int_{-1}^{1} \sqrt{\frac{x-u}{x^{2}-1}} d x, \quad I^{D}=\int_{1}^{u} \sqrt{\frac{x-u}{x^{2}-1}} d x .
$$

Они удовлетворяют уравнениям Пикара-Фукса

$$
\left(\frac{d^{2}}{d u^{2}}+\frac{1}{4\left(u^{2}-1\right)}\right)\left(\begin{array}{c}
I \\
I^{D}
\end{array}\right)=0
$$

которые могут быть использованы для асимптотического разложения действия и препотенциала около точки $u=\infty$ или $u= \pm 1$. $A A$-дуальность проявляется в том, что около $u=\infty$ (отвечающей рассеянию при высокой энергии в квантовой механике и пертурбативному режиму в теории поля) переменной действия является период $I$, а около точки $u=1$ (отвечающей динамике вблизи максимума потенциала в квантовой механике и режиму сильной связи в теории поля) правильная переменная - период $I^{D}$. Инвариантная комбинация периодов [76]

$$
I I^{D}-2 \mathcal{F}=u
$$

может быть выбрана в качестве глобальной координаты на пространстве интегралов движения. При $u \rightarrow \infty$ препотенциал имеет вид

$$
\mathcal{F} \sim \frac{1}{2} u \log u+\cdots \sim I^{2} \log I+\sum_{n} \frac{f_{n}}{n} I^{2-4 n} .
$$

Подчеркнем, что $S$-дуальность отображает динамическую систему в себя, и препотенциал может быть определен для любой многочастичной голоморфной динамической системы.

3.3. Зеркальная симметрия в динамических системах. Рассмотрим еще один тип симметрии, переводяший один класс динамических систем в другие [70, 77, 78]. Напомним, как подобная симметрия формулируется в теории поля. Впервые она была рассмотрена в работе [79] как симметрия, меняющая местами хиггсовскую и кулоновскую ветви пространства модулей, каждая из которых являлась гиперкэлеровым многообразием. Таким образом, симметрия могла быть интерпретирована как гиперкэлерово вращение.

В работе [70] сформулирована обшая процедура, позволяющая описать аналогичную симметрию для интегрируемых систем в рамках метода гамильтоновой редукции. Симметрия переводит координаты одной динамической системы в переменные действия для 
дуальной к ней. Принимая во внимание связь между интегрируемыми многочастичными системами и низкоэнергетическими действиями, можно сформулировать аналог зеркальной симметрии для калибровочных теорий в разном числе измерений.

Качественно симметрию можно описать в разделенных переменных, которые отождествляются с системой точек на 4-мерном гиперкэлеровом многообразии. В наиболее обшем случае естественно рассматривать эллиптически расслоенное многообразие К3. Торический слой определяет импульсы частиц, а торическая база - координаты. Дуальность эффективно меняет местами слой и базу, и соответствуюшая самодуальная динамическая система связана с гипотетической калибровочной теорией с присоединенным гипермультиплетом в шести измерениях. Остальные случаи соответствуют вырождениям дважды эллиптической системы. Вырождение импульсного тора до $\mathbb{C} / \mathbb{Z}_{2}$ отвечает переходу к теории в пяти измерениях, а вырождение до $\mathbb{R}^{2}-$ к 4-мерной теории. Так как комплексный модуль координатного тора играет роль комплексифицированной константы связи, то вырождение координатного тора приводит к подавлению инстантонных эффектов.

Таким образом, мы видим, что зеркальная симметрия связывает калибровочные теории в разном числе измерений. Учет инстантонных эффектов в теории приводит к дополнительной компактификации дуальной к ней теории. В дальнейшем мы будем изучать дуальность классических интегрируемых многочастичных систем, поэтому относительно квантового случая ограничимся лишь кратким комментарием. Так как волновые функции в системах Хитчина оказываются решениями уравнений Книжника-Замолодчикова, то квантовая дуальность означает наличие связи между решениями рациональных тригонометрических и эллиптических задач. Подобная связь была недавно обнаружена [80].

3.3.1. Система двух частиц, соответствующая групше $S U(2)$. Пусть $(p, q)$ - координаты на фазовом пространстве. Дуальный гамильтониан является функцией $q$, выраженной через $I, \varphi$, где $I, \varphi$ - переменные действие - угол исходной системы: $H_{D}(I, \varphi)=$ $H_{D}(q)$. Во всех рассмотренных ниже примерах имеется естественный выбор гамильтониана $H_{D}(q)$.

Рассмотрим в качестве примера эллиптическую модель Калоджеро

$$
H(p, q)=\frac{p^{2}}{2}+\nu^{2} \wp_{\tau}(q),
$$

где переменные $p, q$ комплексны,

$$
\wp_{\tau}(q)=\frac{1}{q^{2}}+\sum_{\substack{(m, n) \in \mathbb{Z}^{2} \\(m, n) \neq(0,0)}} \frac{1}{(q+m \pi+n \tau \pi)^{2}}-\frac{1}{(m \pi+n \tau \pi)^{2}}
$$

- функция Вейерштрасса на эллиптической кривой $E_{\tau}$. Введем обозначения $x=\wp_{\tau}(q)$, $y=\wp_{\tau}(q)^{\prime}$. Уравнение кривой $E_{\tau}$ имеет вид

$$
y^{2}=4 x^{3}-g_{2}(\tau) x-g_{3}(\tau)=4 \prod_{i=1}^{3}\left(x-e_{i}\right), \quad \sum_{i=1}^{3} e_{i}=0 .
$$


Голоморфный дифференциал на кривой $E_{\tau}$ равен $d q=d x / y$. Введем переменную $e_{0}=$ $2 E / \nu^{2}$. Переменная действия совпадает с интегралом от $p d q / 2 \pi$ на кривой $E=H(p, q)$ :

$$
I=\frac{1}{2 \pi} \oint_{A} \sqrt{2\left(E-\nu^{2} \wp_{\tau}(q)\right)}=\frac{1}{4 \pi \mathrm{i}} \oint_{A} \frac{d x \sqrt{x-e_{0}}}{\sqrt{\left(x-e_{1}\right)\left(x-e_{2}\right)\left(x-e_{3}\right)}} .
$$

Угловая переменная определяется из уравнения $d p \wedge d q=d I \wedge d \varphi$ :

$$
d \varphi=\frac{1}{2 \mathrm{i} T(E)} \frac{d x}{\sqrt{\prod_{i=0}^{3}\left(x-e_{i}\right)}},
$$

где $T(E)$ определяет $d \varphi$, так что $A$-период $d \varphi$ равен $2 \pi$ :

$$
T(E)=\frac{1}{4 \pi \mathrm{i}} \oint_{A} \frac{d x}{\sqrt{\prod_{i=0}^{3}\left(x-e_{i}\right)}} .
$$

Таким образом, получаем

$$
\begin{aligned}
2 \mathrm{i} T(E) d \varphi & =\frac{d x}{\sqrt{4 \prod_{i=0}^{3}\left(x-e_{i}\right)}} \\
\omega d \varphi & =\frac{d t}{\sqrt{4 \prod_{i=1}^{3}\left(t-t_{i}\right)}},
\end{aligned}
$$

где

$$
\begin{gathered}
\omega=-2 \mathrm{i} T(E) \sqrt{e_{01} e_{02} e_{03}}=\frac{1}{2 \pi} \oint_{A} \frac{d t}{\sqrt{4 \prod_{i=1}^{3}\left(t-t_{i}\right)}}, \\
t=\frac{1}{x-e_{0}}+\frac{1}{3} \sum_{i=1}^{3} \frac{1}{e_{0 i}}, \quad t_{i}=\frac{1}{3} \sum_{j=1}^{3} \frac{e_{j i}}{e_{0 i} e_{0 j}}, \quad e_{i j}=e_{i}-e_{j} .
\end{gathered}
$$

Определим мероморфную функцию на кривой $E_{\tau}$ :

$$
\widehat{c n}_{\tau}(z)=\sqrt{\frac{x-e_{1}}{x-e_{3}}}
$$

где переменная $z$ имеет периоды $2 \pi$ и $2 \pi \tau$. Тогда гамильтониан имеет вид

$$
H_{D}(I, \varphi)=\widehat{c n}_{\tau}(z)=\widehat{c n}_{\tau_{E}}(\varphi) \sqrt{1-\frac{\nu^{2} e_{13}}{2 E-\nu^{2} e_{3}}},
$$

где $\tau_{E}$ - модулярный параметр спектральной кривой $v^{2}=4 \prod_{i=1}^{3}\left(t-t_{i}\right)$ :

$$
\tau_{E}=\left(\oint_{B} \frac{d t}{\sqrt{4 \prod_{i=1}^{3}\left(t-t_{i}\right)}}\right) /\left(\oint_{A} \frac{d t}{\sqrt{4 \prod_{i=1}^{3}\left(t-t_{i}\right)}}\right) .
$$

При больших $I$ выполняется $2 E(I) \sim I^{2}$. Таким образом, эллиптическая модель Калоджеро с рациональной зависимостью от импульса и эллиптической от координат отображается в систему, эллиптическую по импульсам и рациональную по координате. В калибровочной теории мы связываем модели в четырех и шести измерениях с присоединенной материей. 
3.3.2. Многочастичные системы. Покажем, что зеркальная симметрия естественно формулируется в терминах гамильтоновой и пуассоновой редукций. Она может быть описана, как одновременная смена калибровочных условий и гамильтонианов. Удобно сформулировать дуальности в рациональном и тригонометрическом случаях в следующем виде:

$$
\begin{array}{ccccc} 
& \text { rat.CM } & \leftrightarrow & \text { rat.CM } & \\
R \rightarrow 0 & \uparrow & & \uparrow & \beta \rightarrow 0 \\
& \text { trig.CM } & \leftrightarrow & \text { rat.RS } & \\
\beta \rightarrow 0 & \uparrow & & \uparrow & R \rightarrow 0
\end{array}
$$

Здесь обозначение СМ отвечает моделям Калоджеро-Мозера, a RS - системе Рюсенара-Шнейдера, параметры $R$ и $\beta$ - радиус координатной окружности и величина, обратная скорости света. Стрелки связывают дуальные системы, большая часть которых рассматривалась в [77]. Заметим, что преобразования дуальности образуют группу, которая в случае самодуальных систем содержит $S L_{2}(\mathbb{Z})$. Генератор $S$ задается горизонтальной стрелкой, а генератор $T$ определяет эволюцию системы в дискретном времени.

Рассмотрим пространство $\mathcal{A}_{\mathbf{T}^{2}} S U(N)$-калибровочных полей $A$ на торе $\mathbf{T}^{2}=\mathbf{S}^{1} \times \mathbf{S}^{1}$ с радиусами $R$ и $\beta$. На пространстве $\mathcal{A}_{\mathbf{T}^{2}}$ действует калибровочная группа Клебша-Гордана, сохраняюшая симплектическую форму

$$
\Omega=\frac{k}{4 \pi^{2}} \int \operatorname{Tr} \delta A \wedge \delta A,
$$

где $k$ - произвольное действительное число. Пусть $\left(e_{1}: \ldots: e_{N}\right)$ - однородные координаты на присоединенной орбите $\mathcal{O}$. Тогда отображение момента для действия группы Клебша-Гордана на $\mathcal{A}_{\mathbf{T}^{2}} \times \mathcal{O}$ имеет вид

$$
k F_{A}+J \delta^{2}(x), \quad J_{i j}=\mathrm{i} \nu\left(\delta_{i j}-e_{i} e_{j}^{*}\right)
$$

где $F_{A}-2$-форма кривизны. Мы рассматриваем $e_{i}$ в качестве координат на $\mathbb{C}^{N}$ с условием $\sum_{i}\left|e_{i}\right|^{2}=N$ с точностью до фазового фактора.

Определим набор коммутирующих гамильтонианов. Очевидно, что собственные значения монодромии связности по петле на $\mathbf{T}^{2}$ коммутируют. Рассмотрим редукцию на нулевой уровень отображения момента. Мы имеем, по крайней мере, $N-1$ независимых коммутируюших функций на редуцированном фазовом пространстве $\mathcal{M}_{\nu}$. Найдем размерность $\mathcal{M}_{\nu}$. Если $\nu=0$, то связность плоская и орбиты параметризуются классами сопряженности монодромии по двум нестягиваемым циклам на $\mathbf{T}^{2}-A$ и $B$. Так как фундаментальная группа $\pi_{1}\left(\mathbf{T}^{2}\right) \mathbf{T}^{2}$-абелева, циклы $A$ и $B$ коммутируют. Таким образом, их можно одновременно привести к диагональному виду, и, следовательно, размерность пространства $\mathcal{M}_{0}$ равна $2(N-1)$. В присутствии $\mathcal{O}$ связность является плоской вне $x$ и имеет нетривиальную монодромию вокруг $x$, т.е.

$$
A B A^{-1} B^{-1}=\exp (R \beta J)
$$


Если мы диагонализуем $A$, то $B$ определяется с точностью до умножения справа элементами из группы. Напомним, что орбита $\mathcal{O}$ имеет размерность $2(N-1)$. Редукция $\mathcal{O}$ по отношению к группе состоит из точки и не меняет размерность $\mathcal{M}_{\nu}$. Очевидно, что мы получаем пару дуальных систем, так как в качестве координат мы можем выбирать собственные значения $A$ или $B$.

Двумерное описание подразумевает наличие группы $S L_{2}(\mathbb{Z})$-дуальностей. Операции $S$ и $T$ определены следуюшим образом:

$$
S:(A, B) \mapsto\left(A B A^{-1}, A^{-1}\right), \quad T:(A, B) \mapsto(A, B A),
$$

и связаны с фундаментальной группой двумерного тора. Заметим, что операции $S$ и $T$ сохраняют величину $A B A^{-1} B^{-1}$ и коммутируют с действием калибровочной групшы. Группа Г, генерируемая $S$ и $T$, в пределе $\beta, R \rightarrow 0$ сводится к $S L_{2}(\mathbb{Z})$, так как

$$
A=1+\beta P+\cdots, \quad B=1+R Q+\cdots .
$$

В другом описании мы заменяем пространство двумерных калибровочных полей кокасательным пространством к центрально-расширенной группе петель:

$$
T^{*} \widehat{G}=\left(\mathrm{g}(x), k \bar{\psi}_{x}+P(x)\right) .
$$

Выберем нестягиваемый контур $\mathbf{S}^{1}$ на торе, не проходящий через отмеченную точку $p$. Пусть $x, y$ - координаты на торе, и условие $y=0$ определяет контур $\mathbf{S}^{1}$. Переменная $x$ меняется с периодом $\beta$, а $y$-с периодом $R$. Тогда

$$
P(x)=A_{x}(x, 0), \quad \mathrm{g}(x)=P \exp \left[\int_{0}^{R} A_{y}(x, y) d y\right] .
$$

Отображение момента имеет следующий вид:

$$
k \mathrm{~g}^{-1} \bar{\psi}_{x} \mathrm{~g}+\mathrm{g}^{-1} \mathrm{Pg}-P=J \delta(x),
$$

где $k=1 / R \beta$. Решение в калибровке $P=\operatorname{diag}\left(q_{1}, \ldots, q_{N}\right)$ задает оператор Лакса $A=\mathrm{g}(0)$, причем параметры $R, \beta$ меняются местами. С другой стороны, если мы диагонализуем $\mathrm{g}(x)$ :

$$
\mathrm{g}(x)=\operatorname{diag}\left(z_{1}=e^{\mathrm{i} R q_{1}}, \ldots, z_{N}=e^{\mathrm{i} R q_{N}}\right),
$$

то аналогичным образом получим оператор Лакса

$$
\begin{gathered}
B=P \exp \left[\oint \frac{1}{k} P(x) d x\right]=\operatorname{diag}\left(e^{\mathrm{i} \theta_{i}}\right) \exp (\mathrm{i} R \beta \nu \mathrm{r}), \\
\mathrm{r}_{i j}=\frac{1}{1-e^{\mathrm{i} R q_{j i}}} \quad(i \neq j), \quad \mathrm{r}_{i i}=-\sum_{j \neq i} \mathrm{r}_{i j},
\end{gathered}
$$

что определяет дуальность $A \leftrightarrow B$. Самодуальность тригонометрической модели Рюсенара имеет простую физическую интерпретацию. Модели отвечает теория ЧС в трех измерениях на $\mathbf{T}^{2} \times \mathbb{R}^{1}$ с соответствующими временной вильсоновской линией и пространственной петлей Вильсона. Именно свобода в ориентации последней приводит к наличию дуальных теорий. Группа дуальностей определяется генераторами $S$ и $T$. 


\section{4. СИСТЕМЫ ЧАСТИЦ И СУПЕРСИММЕТРИЧНЫЕ КАЛИБРОВОЧНЫЕ ТЕОРИИ}

Покажем, что рассмотренные выше многочастичные интегрируемые системы возникают при изучении эффективных низкоэнергетических $N=2$ 4-мерных суперсимметричных калибровочных теорий поля. Ниже мы убедимся, что роль многочастичных систем состоит в определении правильных степеней свободы в вакуумном секторе теории. Тот факт, что полный набор данных, описывающих низкоэнергетический сектор теории, задается многочастичной системой, однозначно подразумевает, что число эффективных степеней свободы в вакуумном секторе теории поля конечно. В настоящем разделе мы покажем, каким образом могут быть найдены соответствующие системы частиц, а в разделе 5 найдем подходящие степени свободы в рамках теории струн.

Схема получения эффективного действия предполагает наличие в теории двух масштабов, и эффективное действие возникает как результат интегрирования по “тяжелым" модам. В $N=2$ теории ЯМ подобная схема реализуется следующим образом $[1,2]$. В силу расширенной суперсимметрии в лагранжиане автоматически содержится потенциальньй член для скалярных полей

$$
V(\phi)=\operatorname{Tr}\left[\phi, \phi^{+}\right]^{2}
$$

где след берется по присоединенному представлению калибровочной группы $S U(N)$. Вид потенциала подразумевает наличие долин в теории, вдоль которых энергия вакуума равна нулю и суперсимметрия не нарушена. Скалярное поле в вакуумном состоянии может принимать произвольное значение в подалгебре $\operatorname{Kapтана~} \phi=\operatorname{diag}\left(a_{1}, \ldots, a_{n}\right)$, поэтому можно ввести в теорию набор калибровочно-инвариантных параметров порядка $u_{k}=\left\langle\operatorname{Tr} \phi^{k}\right\rangle$, определяющих конкретное вакуумное состояние.

Так как скалярное поле приобретает вакуумное среднее, то работает стандарный механизм Хиггса, и в области больших значений вакуумного среднего имеются тяжелые калибровочные бозоны. Для получения эффективного низкоэнергетического действия необходимо просуммировать петлевые поправки и мультиинстантонные вклады. Вычисление петель выполнить несложно, так как все поправки, кроме однопетлевой, обрашаются в нуль в силу расширенной суперсимметрии. Явное суммирование по инстантонам выполнить до настояшего времени не удалось, однако дополнительные аргументы позволяют определить эффективное действие без непосредственного суммирования.

Исходное действие $N=2$ теории в $N=2$ суперполях имеет простой вид

$$
S(\Psi)=\operatorname{Im} \tau \int \operatorname{Tr} \Psi^{2}
$$

и требование голоморфности [27] подразумевает, что низкоэнергетическое эффективное действие должно определяться одной функцией, называемой препотенциалом:

$$
S_{\text {eff }}(\Psi)=\operatorname{Im} \int \mathcal{F}(\Psi)
$$


Таким образом, задача сводится к определению всего одной функции, однако согласование действия с принципом дуальности требует введения дополнительных нестандартных объектов в описание теории поля. Трудности, связанные с доказательством дуальности, можно пояснить следующим образом. Если бы теория была $N=4$ суперсимметрична и конечна, то дуальность могла бы быть определена как инвариантность относительно преобразования $\tau \rightarrow \tau^{-1}, \tau \rightarrow \tau+1$, где $\tau=\mathrm{i} / g^{2}+2 \pi \theta$. Однако в асимптотически свободной теории требуется согласование с ренормгруппой и необходимо вводить дополнительные структуры, позволяющие связать преобразование дуальности с модулярными преобразованиями. В работах $[1,2]$ было предложено рассматривать вспомогательные римановы поверхности, матришы периодов которых связаны с эффективными константами связи. С помощью этого приема можно связать преобразование дуальности с модулярными преобразованиями римановой поверхности, которая, например, для $S U(2)$ имеет вид

$$
y^{2}=\left(x^{2}-\Lambda_{\mathrm{QCD}}^{4}\right)(x-u) .
$$

Следуюшим шагом является введение подходяших переменных, модулярные свойства которых согласованы с полевой интерпретацией. Оказалось, что правильными переменными являются интегралы от мероморфных форм по циклам на введенной римановой поверхности. Эти же переменные совпадают с центральными зарядами в алгебре расширенной суперсимметрии. Матрица, составленная из интегралов от форм по циклам, преобразуется относительно действия модулярной группы как сечение расслоения, поэтому ее глобальное поведение, например структура сингулярностей, однозначно определяется данными монодромии.

Преобразование дуальности связывает электрические и магнитные переменные, которые описывают пертурбативные степени свободы в области слабой и сильной связей. Введение римановой поверхности позволяет изучать свойства монодромии дуальной пары переменных при движении в пространстве параметров порядка. В простейшем случае группы $S U(2)$ на плоскости одного параметра порядка $u$ имеются три особые точки, при обходе вокруг которых магнитные и электрические переменные смешиваются друг с другом. Смешивание имеет ясную физическую интерпретацию. В теории с $\theta \neq 0$ монополь приобретает электрический заряд, в то время как поляризация инстантонной среды приводит к появлению индуцированных дионов.

Покажем, каким образом римановы поверхности и мероморфные дифференциалы, определенные на них, возникают при рассмотрении многочастичных интегрируемых систем.

4.1. Цепочка Тоды. Покажем, каким образом данные низкоэнергетического сектора суперсимметричных теорий могут быть связаны с системой частиц. Для теории без материи такой системой частиц является периодическая цепочка Тоды с периодом $N_{c}$ [81-84], уравнения движения для которой имеют вид

$$
\frac{\partial \phi_{i}}{\partial t}=p_{i}, \quad \frac{\partial p_{i}}{\partial t}=e^{\phi_{i+1}-\phi_{i}}-e^{\phi_{i}-\phi_{i-1}} .
$$

Система является интегрируемой, что подразумевает сушествование законов сохранения, число которых равно числу степеней свободы. Для единой записи полного набора 
законов сохранения удобно ввести оператор Лакса, определенный для любой интегрируемой системы. Собственные значения оператора Лакса являются инвариантами динамики, поэтому все интегралы движения являются функциями его собственных значений. Вспомогательная линейная задача для цепочки Тоды имеет вид

$$
\lambda \psi_{n}^{ \pm}=\sum_{k} \mathcal{L}_{n k} \psi_{k}^{ \pm}=e^{\frac{1}{2}\left(\phi_{n+1}-\phi_{n}\right)} \psi_{n+1}^{ \pm}+p_{n} \psi_{n}^{ \pm}+e^{\frac{1}{2}\left(\phi_{n}-\phi_{n-1}\right)} \psi_{n-1}^{ \pm}
$$

причем оператор Лакса коммутирует с оператором сдвига на период $T$ :

$$
T \phi_{n}=\phi_{n+N_{c}}, \quad T p_{n}=p_{n+N_{c}}, \quad T \psi_{n}=\omega \psi_{n+N_{c}}
$$

Ситуация характерна для квантово-механических задач с периодическим потенциалом, в которых для блоховских функций можно ввести квазиимпульс. Соответственно существование двух коммутирующих операторов означает наличие у них общего спектра, связанного соотношением дисперсии. В контексте интегрируемых систем соотношение $\mathcal{P}(\lambda, w)=0$, связывающее собственные значения коммутирующих операторов, определяет риманову поверхность $\Sigma$ и носит название спектральной кривой. Интегралы движения системы могут быть представлены в виде $\operatorname{Tr} L^{k}$ или через разложение матрицы монодромии по спектральному параметру.

В первой формулировке оператор Лакса представляется матрицей $N_{c} \times N_{c}$, зависящей от динамических переменных и собственного значения оператора сдвига $w$, определенного на сфере с двумя отмеченными точками:

$$
\mathcal{L}^{\mathrm{TC}}(w)=\left(\begin{array}{ccccc}
p_{1} & e^{\frac{1}{2}\left(\phi_{2}-\phi_{1}\right)} & 0 & \ldots & w e^{\frac{1}{2}\left(\phi_{1}-\phi_{N_{c}}\right)} \\
e^{\frac{1}{2}\left(\phi_{2}-\phi_{1}\right)} & p_{2} & e^{\frac{1}{2}\left(\phi_{3}-\phi_{2}\right)} & \ldots & 0 \\
0 & e^{\frac{1}{2}\left(\phi_{3}-\phi_{2}\right)} & p_{3} & \ldots & 0 \\
\ldots \ldots \ldots \ldots \ldots \ldots \ldots \ldots \ldots \ldots \ldots \ldots \ldots \ldots \ldots \ldots \ldots \ldots \ldots \ldots & \ldots \ldots \ldots
\end{array}\right) .
$$

Характеристическое уравнение матрицы Лакса

$$
\mathcal{P}(\lambda, w)=\operatorname{det}_{N_{c} \times N_{c}}\left(\mathcal{L}^{\mathrm{TC}}(w)-\lambda\right)=0
$$

определяет спектральную кривую

$$
w+\frac{1}{w}=2 P_{N_{c}}(\lambda)
$$

совпадающую с римановой поверхностью, введенной для описания $S U\left(N_{c}\right)$-теории ЯМ. Интегралы движения динамической системы параметризуют пространство модулей комплексных структур гиперэллиптических поверхностей рода $N_{c}-1$. 
Полезно подробнее рассмотреть случай $S U(2)$, в котором ясна роль параметров суперсимметричной теории. Динамическая система для $S U(2)$ совпадает со стандартным периодическим гамильтонианом

$$
H=p^{2}+\Lambda_{\mathrm{QCD}}^{2} \cos x
$$

Если ввести переменную $y=\sin x$ и предположить, что переменные на фазовом пространстве комплексны, то уравнение римановой поверхности в суперсимметричной теории совпадает с уравнением, определяюшим поверхность уровня гамильтониана при энергии $H=u$. Таким образом, мы видим, что параметр порядка суперсимметричной теории играет роль гамильтониана, а $\Lambda_{\mathrm{QCD}}$ - роль константы связи. Пертурбативный режим в теории поля соответствует области высокой энергии, а область сильной связи эквивалентна области энергии, близкой к амплитуде потенциала.

В альтернативной формулировке вводятся локальные операторы на каждом узле цепочки

$$
L_{i}^{\mathrm{TC}}(\lambda)=\left(\begin{array}{cc}
p_{i}+\lambda & e^{\phi_{i}} \\
e^{-\phi_{i}} & 0
\end{array}\right), \quad i=1, \ldots, N_{c}
$$

и соответственно полная матрица монодромии

$$
T_{N_{c}}(\lambda)=\prod_{N_{c} \geqslant i \geqslant 1} L_{i}(\lambda)
$$

характеристическое уравнение для которой вновь определяет спектральную кривую

$$
\operatorname{det}_{2 \times 2}\left(T_{N_{c}}^{\mathrm{TC}}(\lambda)-w\right)=w^{2}-w \operatorname{Tr} T_{N_{c}}^{\mathrm{TC}}(\lambda)+\operatorname{det} T_{N_{c}}^{\mathrm{TC}}(\lambda)=w^{2}-w \operatorname{Tr} T_{N_{c}}^{\mathrm{TC}}(\lambda)+1=0,
$$

или

$$
\mathcal{P}(\lambda, w)=\operatorname{Tr} T_{N_{c}}^{\mathrm{TC}}(\lambda)-w-\frac{1}{w}=2 P_{N_{c}}(\lambda)-w-\frac{1}{w}=0 .
$$

Построение мероморфных дифференциалов на спектральных поверхностях в терминах интегрируемых систем мы обсудим ниже.

4.2. Теория с гипермультиплетом в присоединенном представлении и системы Калоджеро. Рассмотрим простейшую деформацию $N=2$ суперсимметричной теории, полученную добавлением гипермультиплета в присоединенном представлении [85-89]. Если гипермультиплет безмассовый, то теория конечна и имеет константу связи, определенную на любом масштабе. Мы будем рассматривать в основном массивный гипермультиплет и найдем соответствующую динамическую систему, обладаюшую дополнительным параметром. Оказывается, что деформация массивным гипермультиплетом может быть описана в терминах эллиптической модели Калоджеро. При этом масса гипермультиплета играет роль константы связи в модели Калоджеро, а ультрафиолетовая константа связи в теории поля определяет модуль эллиптической кривой, на которой вводится спектральный параметр. Процедура размерной трансмутации в теории поля имеет конечномерный аналог и эквивалентна переходу от системы Калоджеро к системе Тоды. 
Матричный $\left(N_{c} \times N_{c}\right)$-оператор для системы Калоджеро был найден в разделе 3 и имеет вид

$$
\begin{aligned}
& \mathcal{L}^{\mathrm{Cal}}(\xi)=\left(\mathbf{p H}+\sum F(\mathbf{q} \mid \xi) E\right)=
\end{aligned}
$$

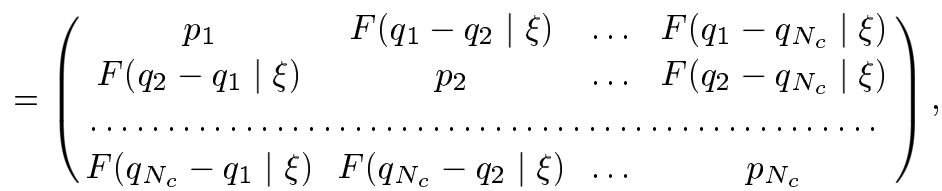

где константа связи пропорциональна массе гипермультиплета $\mathrm{m}^{2}$.

Спектральная кривая $\Sigma^{\mathrm{Cal}}$

$$
\operatorname{det}_{N_{c} \times N_{c}}\left(\mathcal{L}^{\mathrm{Cal}}(\xi)-\lambda\right)=0
$$

накрывает затравочную спектральную кривую $E(\tau)$

$$
y^{2}=\left(x-e_{1}\right)\left(x-e_{2}\right)\left(x-e_{3}\right) .
$$

Редукция к системе Тоды описывается следующим образом $[68,87,89]$. Предполагается, что переменные $\tau_{0}$ и $M$ стремятся к бесконечности, а

$$
q_{i}-q_{j}=\left[(i-j) \log m+\left(\phi_{i}-\phi_{j}\right)\right],
$$

поэтому вместо затравочной константы связи $\tau$ вводится перенормированная константа связи, а масштабный параметр возникает из соотношения $\Lambda^{N_{c}} \sim m^{N_{c}} \exp (\mathrm{i} \pi \tau)$. В этом пределе эллиптическая кривая вырождается в сферу с координатами $w=e^{\xi} \exp (\mathrm{i} \pi \tau)$.

Простейшим примером спектральной кривой для модели Калоджеро с $N_{c}=2$ является кривая второго рода. Действительно, в этом случае уравнение (4.13) можно переписать в виде

$$
\mathcal{P}(\lambda ; x, y)=\lambda^{2}-h_{2}+\frac{g^{2}}{\omega^{2}} x=0,
$$

что соответствует двукратному накрытию эллиптической кривой $E(\tau)$, которое может быть представлено как два тора, склеенные вдоль одного разреза. Таким образом, в случае $N_{c}=2$ кривая является гиперэллиптической.

4.3. Уравнения Уизема. Для определения низкоэнергетических лагранжианов, кроме спектральных кривых, требуется получение препотенциала. Простейшая процедура получения препотенциала состоит в разрешении соотношения $a_{D}=\partial \mathcal{F} / \partial a$, так как для любых переменных $a, a_{D}$ имеется представление в виде интегралов от мероморфных дифференциалов по спектральной кривой. Оказывается, что они допускают интерпретацию в терминах переменных действия в системах Калоджеро и Тоды.

Рассмотрим обшую процедуру введения производящего дифференциала в интегрируемых системах [4]. Она основана на аналоге ренормгрупповых потоков в теории поля. 
В теории поля производится интегрирование по “тяжелым" модам, после чего возникает эффективное действие для “легких" мод. В теории интегрируемых систем аналогичная процедура выглядит следующим образом. Рассматриваются решения классических уравнений движения, обладающие набором параметров, соответствующих коллективным координатам (модулям). На следуюшем шаге предполагается, что модули адиабатически зависят от пространственно-временных переменных, причем временем в медленной эволюции является ренормгрупповой масштаб $\log \Lambda_{\mathrm{QCD}}$. Таким образом, мы имеем динамику Уизема на пространстве модулей, для которой производящий дифференциал играет роль дифференциала действия, а препотенциал является логарифмом $\tau$-функции. Для конечнозонных решений, которые ответственны за описание суперсимметричных калибровочных теорий, в качестве модулей естественно выбирать точки ветвления соответствуюших гиперэллиптических кривых.

Дифференциал $d S(z)$ может быть построен для любых конечнозонных решений, он совпадает с производящей формой в теории поля. Соотношение

$$
\frac{\partial \mathcal{F}}{\partial \mathbf{a}}=\mathbf{a}_{D}, \quad \mathbf{a}=\oint_{\mathbf{A}} d S, \quad \mathbf{a}_{D}=\oint_{\mathbf{B}} d S,
$$

определяет $\tau$-функцию иерархии Уизема $\mathcal{F}=\log \mathcal{T}_{\text {Whitham }}[90]$.

Рассмотрим динамику Уизема на примере решения Виттена-Зайберга для группы $S U(2)$. В качестве динамической переменной естественно рассмотреть точки ветвления эллиптической кривой. Решение, представляюшее наибольший интерес, было найдено Гуревичем и Питаевским [91] в контексте гидродинамики плазмы и соответствует движению лишь одной точки ветвления при фиксированных положениях остальных. В качестве времени в динамической системе выступает величина $\log \Lambda_{\mathrm{QCD}}$. Явное решение имеет вид

$$
\begin{aligned}
U\left(t_{1}, t_{3}, \ldots \mid u\right) & =\frac{\partial^{2}}{\partial t_{1}^{2}} \log \mathcal{T}\left(t_{1}, t_{3}, \ldots \mid u\right)= \\
& =U_{0} \wp\left(k_{1} t_{1}+k_{3} t_{3}+\cdots+\Phi_{0} \mid \omega, \omega^{\prime}\right)+\frac{u}{3}
\end{aligned}
$$

Удобно ввести дифференциалы

$$
d p \equiv d \Omega_{1}(z)=\frac{z-\alpha(u)}{y(z)} d z, \quad d Q \equiv d \Omega_{3}(z)=\frac{z^{2}-\frac{1}{2} u z-\beta(u)}{y(z)} d z
$$

с условиями нормировки

$$
\alpha(u)=\oint_{A} \frac{z d z}{y(z)} / \oint_{A} \frac{d z}{y(z)}, \quad \beta(u)=\oint_{A} \frac{\left(z^{2}-\frac{1}{2} u z\right) d z}{y(z)} / \oint_{A} \frac{d z}{y(z)} .
$$

Соответствуюший решению производяший дифференциал, если $t_{k>1}=0$, имеет вид [81]

$$
d S(z)=\left(t_{1}+t_{3}\left(z+\frac{1}{2} u\right)+\cdots\right) \frac{z-u}{y(z)} d z=t_{1} \frac{z-u}{y(z)} d z .
$$

2 Теоретическая и математическая физика, т. 125, № 1, 2000 г. 
Из (4.21) получаем

$$
\begin{aligned}
& \frac{\partial d S(z)}{\partial t_{1}}=\left(z-u-\left(\frac{1}{2} t_{1}+u t_{3}\right) \frac{\partial u}{\partial t_{1}}\right) \frac{d z}{y(z)} \\
& \frac{\partial d S(z)}{\partial t_{3}}=\left(z^{2}-\frac{1}{2} u z-\frac{1}{2} u^{2}-\left(\frac{1}{2} t_{1}+u t_{3}\right) \frac{\partial u}{\partial t_{3}}\right) \frac{d z}{y(z)}, \ldots
\end{aligned}
$$

и, сравнивая с явными выражениями, имеем

$$
\left(\frac{1}{2} t_{1}+u t_{3}\right) \frac{\partial u}{\partial t_{1}}=\alpha(u)-u, \quad\left(\frac{1}{2} t_{1}+u t_{3}\right) \frac{\partial u}{\partial t_{3}}=\beta(u)-\frac{1}{2} u^{2} .
$$

Таким образом, решение Гуревича-Питаевского имеет структуру [91]

$$
\frac{\partial u}{\partial t_{3}}=v_{31}(u) \frac{\partial u}{\partial t_{1}}
$$

где

$$
v_{31}(u)=\frac{\beta(u)-\frac{1}{2} u^{2}}{\alpha(u)-u}=\left.\frac{d \Omega_{3}(z)}{d \Omega_{1}(z)}\right|_{z=u},
$$

и выражается в эллиптических функциях. Действие, вычисленное на решении Гуревича-Питаевского, определяет препотенциал в решении Виттена-Зайберга. Обобщение динамики Уизема на случай калибровочной группы произвольного ранга было найдено в работах $[83,84]$.

4.4. Суперсимметричная $\mathbf{K X} Д$ и $X X X$-спиновые цепочки. Перейдем к рассмотрению теорий с фундаментальными гипермультиплетами материи с произвольными массами. Согласно условию асимптотической свободы мы будем предполагать, что $N_{f} \leqslant 2 N_{c}$. Как и для недеформированной калибровочной модели, мы найдем интегрируемую систему, которая содержит всю информацию про низкоэнергетический сектор теории. Оказьвается, что подходяшей интегрируемой системой является неоднородная спиновая цепочка, а в предельном случае $N_{f}=2 N_{c}$ - спиновая цепочка в магнитном поле [92, 93].

Для получения спектральной кривой нам потребуется выражение для $(2 \times 2)$-оператора Лакса в $s l(2) X X X$-спиновой цепочке

$$
L(\lambda)=\lambda \cdot \mathbf{1}+\sum_{a=1}^{3} S_{a} \cdot \sigma^{a}
$$

Скобки Пуассона динамических переменных $S_{a}, a=1,2,3$, задаются рациональной $r$-матрицей

$$
r(\lambda)=\frac{1}{\lambda} \sum_{a=1}^{3} \sigma^{a} \otimes \sigma^{a}
$$

В случае $s l(2)$ они имеют вид

$$
\left\{S_{a}, S_{b}\right\}=\mathrm{i} \epsilon_{a b c} S_{c},
$$


т.е. элементы $\left\{S_{a}\right\}$ играют роль углового момента. Алгебра обладает оператором Казимира

$$
K^{2}=\mathbf{S}^{2}=\sum_{a=1}^{3} S_{a} S_{a}
$$

поэтому

$$
\begin{gathered}
\operatorname{det}_{2 \times 2} L(\lambda)=\lambda^{2}-K^{2}, \\
\operatorname{det}_{2 \times 2} T_{N_{c}}(\lambda)=\prod_{i=1}^{N_{c}} \operatorname{det}_{2 \times 2} L_{i}\left(\lambda-\lambda_{i}\right)=\prod_{i=1}^{N_{c}}\left(\left(\lambda-\lambda_{i}\right)^{2}-K_{i}^{2}\right)= \\
=\prod_{i=1}^{N_{c}}\left(\lambda+m_{i}^{+}\right)\left(\lambda+m_{i}^{-}\right),
\end{gathered}
$$

где мы предположили, что спины $K$ принимают в узлах различные значения и задают значения масс фундаментальных гипермультиплетов в суперсимметричной КХД

$$
m_{i}^{ \pm}=-\lambda_{i} \mp K_{i}
$$

Безмассовому пределу отвечает случай однородной цепочки $\lambda_{i}=0$ с нулевым спином в каждом узле $K_{i}=0$.

Детерминант полной матрицы монодромии зависит от вакуумных средних скалярного поля в суперсимметричной КХД только через значения операторов Казимира в узлах, а величина $\mathcal{T}_{N_{c}}(\lambda)=\left(\operatorname{Tr}_{2 \times 2} T_{N_{c}}(\lambda)\right) / 2$ зависит от модулей кулоновской ветви только через гамильтонианы спиновой цепочки.

Специального рассмотрения требует случай $N_{f}=2 N_{c}$, который для безмассовых фермионов соответствует конечной теории. С точки зрения интегрируемости необходимо рассмотреть цепочку во внешнем магнитном поле, значение которого оказывается связанным с затравочной константой связи [93].

Технически введение магнитного поля в спиновую цепочку достигается наложением нетривиальных граничных условий с помощью матрицы $V^{-1}$. Кривая для случая $N_{f}=$ $2 N_{c}$ была найдена в работах $[2,94]$ :

$$
\begin{gathered}
w-\frac{Q(\lambda)}{w}=P(\lambda), \quad P(\lambda)=\prod_{i=1}^{n}\left(\lambda-\phi_{i}\right), \\
Q(\lambda)=h(h+1) \prod_{j=1}^{2 n}\left(\lambda-m_{j}-\frac{2 h}{n} \sum_{i} m_{i}\right), \quad h(\tau)=\frac{\theta_{2}^{4}}{\theta_{4}^{4}-\theta_{2}^{4}},
\end{gathered}
$$

где $\tau$ определяется затравочной константой связи, а $\theta_{i}$ - тэта-константы.

Подобная кривая может быть получена, если ввести следующие матрицы, задаюшие граничные условия:

$$
U_{i}=\left(\begin{array}{cc}
1 & 0 \\
0 & \alpha_{i}
\end{array}\right), \quad V=\left(\begin{array}{cc}
1 & h(h+1) \\
\prod_{i} \alpha_{i} & 0
\end{array}\right),
$$


т.е.

$$
V^{-1}=\frac{1}{\operatorname{det} V}\left(\begin{array}{cc}
0 & h(h+1) \\
\prod_{i} \alpha_{i} & -1
\end{array}\right)
$$

Размерная трансмутация, приводящая к появлению массивного параметра в инфракрасной области, соответствует устремлению магнитного поля и массы фермиона к бесконечности при конечном ренорминвариантном параметре $\Lambda_{\mathrm{QCD}}$.

Заметим, что интерпретация масс фундаментальной материи как координат в пространстве полей может быть пояснена следуюшим образом. Рассмотрим свободную частицу в 4-мерном пространстве с законом дисперсии

$$
E^{2}=\vec{p}^{2}+m^{2}
$$

и заметим, что в комплексном импульсном пространстве имеется пересечение уровней в точке $\vec{p}=0$ в безмассовом случае или на поверхности $p^{2}=-m^{2}$ при ненулевой массе. Согласно обшей логике в контексте фазы Берри в пространстве импульсов, рассмотренном как пространство параметров, в точках пересечения уровней расположены монополи, а на поверхности пересечения уровней - “дефекты” более высокой коразмерности.

Таким образом, масса действительно определяет положение особенностей в импульсном пространстве. В разделе 5 мы увидим, что в точках пространства нулевых гармоник скалярного поля с координатами, равными массам, расположены браны разных размерностей. Это согласуется с приведенными выше аргументами, если учесть, что в силу калибровочной инвариантности импульсы и калибровочные поля входят в уравнения движения в виде ковариантной производной.

Наличие монопольной сингулярности в точке $\vec{p}=0$ в безмассовом случае приводит к тому, что оператор вращений в импульсном пространстве имеет дополнительное слагаемое - явление, хорошо известное для операторов врашения в поле монополя. Более того, монопольное слагаемое в операторе момента позволяет определить преобразование киральности для частиц спинов 0,1 и 2 в теории без взаимодействия как врашение по замкнутому контуру в импульсном пространстве. В теории с взаимодействием преобразование киральности в общем случае аномально, поэтому столь простые аргументы не применимы. Однако оказывается, что явление аномалии само может быть описано в терминах возникновения фазы Берри в пространстве полей в случае внутренних и внешних аномалий. Вновь мы имеем качественно удовлетворительное согласие, так как киральная инвариантность нарушается либо явно массой, либо аномалией: в обоих случаях нарушение связано с появлением особенностей в “обобшенном импульсном пространстве".

Упомянем еще одно обобщение, при котором калибровочная группа теории представляет собой произведение нескольких $S U(n)$-факторов

$$
G=\prod_{i=1}^{p-1} S U\left(n_{i}\right)
$$

В теорию могут быть также включены $p-2$ массивных гипермультиплета в представлениях $\left(n_{i}, \bar{n}_{i+1}\right)$. Теории такого типа были рассмотрены в бранном контексте 
в работе [42], где были найдены спектральные кривые, задающие низкоэнергетическую теорию, а соответствуюшими интегрируемыми системами являются высшие магнетики [93].

4.5. Твистованная неоднородная $X X Z$-спиновая цепочка и 5-мерные теории. Опишем интегрируемую теорию, соответствующую 5-мерной теории, - неоднородную $X X Z$-спиновую цепочку - и определим, пользуясь информацией, полученной из интегрируемости, спектральную кривую и производящий дифференциал [93]. В рамках теории поля 5-мерные теории подробно обсуждались в работах $[95,96]$.

Матрища Лакса для $X X Z(S L(2))$-магнетика имеет вид

$$
L(\mu)=\left(\begin{array}{cc}
\mu e^{S_{0}}-\mu^{-1} e^{-S_{0}} & 2 S_{-} \\
2 S_{+} & \mu e^{-S_{0}-\mu^{-1} e^{S_{0}}}
\end{array}\right) .
$$

Нетрудно понять, что спектральный параметр определен на цилиндре, поэтому естественно ввести переменную $\xi: \mu=e^{\xi}$, т.е. оператор Лакса тригонометричен и сплетается $r$-матрицей

$$
r(\xi)=\frac{\mathrm{i}}{\operatorname{sh} \pi \xi}\left(\sigma_{1} \otimes \sigma_{1}+\sigma_{2} \otimes \sigma_{2}+\operatorname{ch} \pi \xi \sigma_{3} \otimes \sigma_{3}\right),
$$

задающей скобку Пуассона в магнетике

$$
\left\{L(\mu) \stackrel{\otimes}{,} L\left(\mu^{\prime}\right)\right\}=\left[r\left(\mu-\mu^{\prime}\right), L(\mu) \otimes L\left(\mu^{\prime}\right)\right]
$$

и алгебру операторов $S_{i}$

$$
\left\{S_{+}, S_{0}\right\}= \pm S_{ \pm}, \quad\left\{S_{+}, S_{-}\right\}=\operatorname{sh} 2 S_{0}
$$

Второй оператор Казимира в алгебре имеет вид

$$
C_{2}=\operatorname{ch} 2 S_{0}+2 S_{+} S_{-}
$$

Нелинейные коммутационные соотношения (4.38) определяют квантовую алгебру $U_{q}(S L(2))$, которая обобщает классическую алгебру $S L(2)$, являющуюся алгеброй симметрии $X X X$-магнетика. Параметром квантовой деформации является радиус пятого измерения $R_{5}$, причем зависимость от радиуса может быть легко восстановлена заменой $S_{i}$ на $R_{5} S_{i}$

Мы вводим матрицу перехода $i$ к $i+n$ соотношением

$$
T(\mu) \equiv L_{n}(\mu) \ldots L_{1}(\mu)
$$

в терминах которой спектральная кривая имеет вид

$$
\operatorname{det}(T(\mu)+w \cdot \mathbf{1})=0
$$

и генерирует полный набор интегралов движения. 
Спектральная кривая для $X X Z$-магнетика может быть приведена к виду

$$
w+\frac{Q_{2 N_{c}}\left(e^{2 \xi}\right)}{e^{2 N_{c}\left(\xi-\xi_{i}\right)} w}=e^{-N_{c}\left(\xi-\xi_{i}\right)} P_{N_{c}}\left(e^{2 \xi}\right)
$$

$\mathrm{C}$ помошью замены переменных $e^{2 \xi}=\mu^{2} \equiv \lambda, w \rightarrow e^{-N_{c}\left(\xi-\xi_{i}\right)} w$ она приводится к гиперэллиптическому виду:

$$
w+\frac{Q_{2 N_{c}}(\lambda)}{w}=P_{N_{c}}(\lambda), \quad Y^{2}=P_{N_{c}}^{2}(\lambda)-4 Q_{N_{c}}(\lambda), \quad Y \equiv w-\frac{Q_{2 N_{c}}(\lambda)}{w}
$$

где полином

$$
Q_{2 N_{c}}(\lambda)=\prod_{i}\left(\lambda^{2}-2 C_{2, i} e^{-\xi_{i}} \lambda+e^{-2 \xi_{i}}\right) \equiv \prod_{i}\left(\lambda-e^{m_{i}^{(+)}}\right)\left(\lambda-e^{m_{i}^{(-)}}\right)
$$

определяет массы фундаментальных гипермультиплетов $m_{i}^{( \pm)}$, которые являются функциями оператора Казимира и неоднородности в соответствующем узле $C_{2, i}$, а полином $P_{N_{c}}(\lambda)$ определяет зависимость от кулоновских модулей вакуума в 5-мерной теории.

Для вырождения теории с материей в чисто калибровочную теорию необходимо воспользоваться симметрией задачи и сделать преобразование оператора Лакса. Представим повернутую матрищу Лакса в виде

$$
L(\mu)=\left(\begin{array}{cc}
\mu A^{(+)}+\mu^{-1} A^{(-)} & B \\
C & \mu D^{(+)}+\mu^{-1} D^{(-)}
\end{array}\right),
$$

совместном с $r$-матрицей (4.36). Для проведения предельного перехода к релятивистской системе Тоды умножим оператор на функцию $e^{S_{0}}$ :

$$
L(\mu)=\left(\begin{array}{cc}
\mu e^{2 S_{0}}-\rho \mu^{-1} & 2 S_{-} e^{S_{0}} \\
2 S_{+} e^{S_{0}} & \theta \mu-\nu \mu^{-1} e^{2 S_{0}}
\end{array}\right)
$$

и выберем параметры поворота в виде

$$
\theta=\nu=0, \quad \rho=1,
$$

т.е.

$$
L(\mu)=\left(\begin{array}{cc}
\mu e^{2 S_{0}}-\mu^{-1} & 2 S_{-} e^{S_{0}} \\
2 S_{+} e^{S_{0}} & 0
\end{array}\right)
$$

Согласно коммутационным соотношениям $\left\{S_{+}, S_{-}\right\}=0$ алгебра может быть реализована в виде $S_{ \pm}=e^{ \pm q}, S_{0}=q, \quad\{p, q\}=1$, а оператор Лакса сводится к оператору для релятивистской системы Тоды, описьваюшей чисто калибровочную 5 -мерную теорию [62]. Автоматически воспроизводится и правильная спектральная кривая. 
Описание калибровочной теории в терминах спиновой цепочки допускает обобщение на случай произведения групп. Как и в 4-мерной теории, необходимо рассмотреть высшие $X X Z$-магнетики типа $S L(p)$ с оператором Лакса

$$
\begin{gathered}
L(\mu)=\sum_{i, j} e_{i j} \otimes L_{i j}, \\
L_{i i}=\mu e^{S_{0, i}}-\mu^{-1} e^{-S_{0, i}}, \quad L_{i j}=2 S_{j i} \quad(i \neq j) .
\end{gathered}
$$

Здесь переменные $S_{0, i}$ связаны с векторами $\epsilon_{i}$, определяющими простые корни $\alpha_{i}=\epsilon_{i}-$ $\epsilon_{i+1}$, а матрица $e_{i j}$ имеет единственный ненулевой матричньй элемент $(i, j)$.

С помощью (4.49) можно получить спектральную кривую

$$
w^{p}+\frac{Q_{N_{c}}^{(1)}\left(\mu^{2}\right)}{\mu^{2 N_{c}}} w^{p-1}+\cdots+\frac{Q_{k N_{c}}^{(k)}\left(\mu^{2}\right)}{\mu^{2 k N_{c}}} w^{p-k}+\cdots+\frac{Q_{p N_{c}}^{(p)}\left(\mu^{2}\right)}{\mu^{2 p N_{c}}}=0
$$

которая согласована с общей бранной конфигурацией из работы [97]. Пертурбативный дифференциал $d S$ имеет вид

$$
d S=\log \lambda d \log \left(\frac{P(\lambda)}{\sqrt{Q(\lambda)}}\right)
$$

что для $S U\left(N_{c}\right)$-теории без материи дает $[62,26]$

$$
\begin{aligned}
\mathcal{F} & =\frac{1}{4} \sum_{i, j}\left(\frac{1}{3}\left|a_{i j}^{3}\right|-\frac{1}{2} \operatorname{Li}_{3}\left(e^{-2\left|a_{i j}\right|}\right)\right)+\frac{N_{c}}{2} \sum_{i>j>k} a_{i} a_{j} a_{k}= \\
& =\frac{1}{4} \sum_{i, j}\left(\frac{1}{3}\left|a_{i j}^{3}\right|-\frac{1}{2} \operatorname{Li}_{3}\left(e^{-2\left|a_{i j}\right|}\right)\right)+\frac{N_{c}}{6} \sum_{i} a_{i}^{3} .
\end{aligned}
$$

В теории с фундаментальной материей получаем [93, 98]

$$
\begin{aligned}
F= & \frac{1}{4} \sum_{i, j} f^{(5)}\left(a_{i j}\right)-\frac{1}{4} \sum_{i, \alpha} f^{(5)}\left(a_{i}+m_{\alpha}\right)+\frac{1}{16} \sum_{\alpha, \beta} f^{(5)}\left(m_{\alpha}-m_{\beta}\right)+ \\
& +\frac{1}{12}\left(2 N_{c}-N_{f}\right)\left(\sum_{i} a_{i}^{3}+\frac{1}{4} \sum_{\alpha} m_{\alpha}^{3}\right),
\end{aligned}
$$

где мы ввели функции $f^{(d)}(x)$ :

$$
f^{(4)}(x)=x^{2} \log x, \quad f^{(5)}(x)=\left(\frac{1}{3}\left|x^{3}\right|-\frac{1}{2} \operatorname{Li}_{3}\left(e^{-2|x|}\right)\right) .
$$

Эффективная константа связи имеет вид $\log \left(\operatorname{sh} a R_{5}\right)$ и может быть получена из второй производной препотенциала в 4-мерной теории (равной $\log a)$ суммированием по модам Калуцы-Клейна:

$$
\log \left(\operatorname{sh} a R_{5}\right)=\sum_{m} \log \left(a+\frac{m}{R_{5}}\right)
$$


Заметим, что описание, следующее из анизотропных спиновых цепочек, согласуется с картиной, полученной в М-теории [99].

Интересно рассмотреть предельные переходы в 4-мерные и 5-мерные теории без материи. Переход к случаю $d=4$ отвечает пределу $R_{5} \rightarrow \infty$. В этом пределе $f^{(5)}(x) \underset{x \sim 0}{\longrightarrow}$ $f^{(4)}(x)$, и мы воспроизводим $4 d$ препотенциал. Другим пределом является плоская 5 -мерная теория. В этом случае отличны от нуля только кубические члены в выражении (4.54):

$$
\begin{aligned}
F= & \frac{1}{12} \sum_{i, j}\left|a_{i j}\right|^{3}-\frac{1}{12} \sum_{i, \alpha}\left|a_{i}+m_{\alpha}\right|^{3}+\frac{1}{48} \sum_{\alpha, \beta}\left|m_{\alpha}-m_{\beta}\right|+ \\
& +\frac{1}{12}\left(2 N_{c}-N_{f}\right)\left(\sum_{i} a_{i}^{3}+\frac{1}{4} \sum_{\alpha} m_{\alpha}^{3}\right),
\end{aligned}
$$

которые согласно [95] можно идентифицировать с членами, возникающими из лагранжиана ЧС. Они могут быть затравочными, а также возникать при вычислении петлевых диаграмм.

В формуле (4.54) граница насышается при $c_{\mathrm{cl}}=N_{c}-N_{f} / 2$, причем интегрируемость допускает разные значения параметра. Действительно, выбирая подходящие значения операторов Казимира на $m$ узлах решетки $\lambda^{m} \bar{Q}_{N_{f}}(\lambda)$, нетрудно получить, что $c_{\mathrm{cl}}=$ $N_{c}-m-N_{f} / 2$. Учитывая целочисленность $m$ и очевидное неравенство $m+N_{f} \geqslant 2 N_{c}$, мы получаем рассмотренное выше ограничение на $c_{\mathrm{cl}}$.

Можно найти интегрируемую систему, отвечающую 6 -мерным теориям с двумя компактными измерениями. Оказывается, что 6-мерная теория с фундаментальной материей описывается неоднородной $X Y Z$-спиновой цепочкой с $N_{c}$ узлами [93]. Любая 6-мерная теория связана с эллиптической кривой, определенной в двух компактных измерениях. Комплексньй модуль этой кривой может быть отождествлен со структурной константой алгебры Склянина. Последняя, как известно, определяет группу симметрии $X Y Z$-спиновой цепочки. В п. 4.4 в 5-мерных теориях мы использовали вырожденную версию алгебры Склянина - квантовую группу с параметром $q=\exp \left(-R_{5}\right)$.

Заметим, что наиболее общая из рассматриваемого класса интегрируемая система соответствует случаю, при котором координаты и импульсы определены на двух независимых торах. В 5-мерной теории без присоединенной материи мы исследовали вырожденньй случай, когда координаты и импульсы были определены на цилиндрах, а в 6-мерной теории импульсы интегрируемой системы определены на торе с радиусами $R_{5}, R_{6}$, а координаты остаются заданными на цилиндре.

\section{5. БРАНЫ КАК СТЕПЕНИ СВОБОДЫ ПРИ НИЗКИХ ЭНЕРГИЯХ В СУПЕРСИММЕТРИЧНЫХ КАЛИБРОВОЧНЫХ ТЕОРИЯХ}

5.1. Теории поля на мировой поверхности D-браны. Начнем с описания непертурбативных состояний в теории струн, которые играют ключевую роль при описании степеней свободы в низкоэнергетическом секторе суперсимметричных калибровочных теорий. Покажем, что фазовое пространство интегрируемых систем, рассмотрен- 
ных в предыдущем разделе, задается коллективными координатами бран различной размерности. Более того, непертурбативные дуальности в теории струн позволяют предложить несколько эквивалентных формулировок одной и той же теории поля.

Центральными идеями, привнесенными в теорию струн в результате точного решения $N=2$ теории ЯМ, оказались дуальность между режимами сильной и слабой связи в теории и возможность нахождения точных формул для масс солитонных состояний, удовлетворяюших условию БПС. Оказалось, что для согласованного описания дуальности в теории струн необходимо вводить солитонные объекты различной размерности - $p$-браны, протяженные в $p$-пространственных измерениях $[33,34]$. Классически их массы ведут себя как $m=g^{-2}$, где $g$ - константа связи, причем формулы для масс БПС-состояний, связанные с центральными зарядами алгебры расшшренной суперсимметрии, остаются точными и на квантовом уровне. В режиме, при котором константа связи велика, солитоны становятся легчайшими объектами в теории и определяют динамику модели в этой области. При этом оказывается возможным сделать переход к дуальным переменным, после чего теория оказывается слабосвязанной в терминах солитонных полей.

Преобразование дуальности хорошо определено в конечной теории, как, например, дуальность Монтонена-Олива в теории с $N=4$ суперсимметрией. В $N=2$ теориях, которые будут обсуждаться ниже, дуальность формулируется в терминах специальной римановой поверхности, определяющей БПС-спектр модели. Появление римановой поверхности в дальнейшем будет объяснено на языке бран. Именно она в большинстве случаев оказывается частью мировой поверхности браны некоторой размерности. Структура пересечений бран фиксируется требованиями суперсимметрии, и набор возможных конфигураций, определяюших теорию поля, оказывается конечным или даже единственным.

Ключевым обстоятельством, позволяющим использовать браны для построения калибровочных теорий поля, является присутствие $U(1)$-калибровочного поля на мировой поверхности браны. Известно, что браны несут заряд в рамон-рамоновском секторе теории $[33,34]$ и открытые струны могут на них заканчиваться. Эти свойства бран позволили Виттену сформулировать новый взгляд на калибровочную теорию как теорию, определенную на мировой поверхности совпадаюших бран, каждая из которых несет на мировой поверхности $U(1)$-калибровочную группу. Открытые струны, соединяющие отдельные браны, делают теорию неабелевой, причем ранг калибровочной группы определяется числом совпадающих бран. В дальнейшем мы будем следовать именно этой логике: суперсимметричные теории поля будут определены на бранной мировой поверхности, а все параметры теории вводятся через геометрию бранных конфигураций.

Основными параметрами в $N=2$ теориях являются координаты на кулоновской ветви пространства модулей - конденсаты скалярных полей, затравочные массы и константа связи. В терминах бран они задаются следующим образом: вакуумные значения скалярных полей определяют вложение бран в объемлющее пространство, константа связи задается расстоянием между дополнительными (по отношению к тем, на которых опре- 
делена теория) бранами, а масса фермионов - это координата соответствующих бран в подходящем измерении. Ниже мы приведем различные примеры, в которых бранная картина будет вполне конкретна, а координаты бран в объемлюшем пространстве оказываются степенями свободы в конечномерной интегрируемой системе, определяюшей низкоэнергетическое действие в суперсимметричной калибровочной теории.

Подчеркнем, что калибровочные теории Хитчина, ответственные за появление интегрируемых многочастичных систем, непосредственно не связаны с суперсимметричными теориями поля в 4-мерном пространстве. Они возникают в “импульсном" или “полевом" пространствах, и в качестве простейшего примера, поясняющего характер их происхождения, можно привести D0-браны на многообразии $T^{4}$. Если сделать преобразование $T$-дуальности по всем координатам, то возникнет суперсимметричная калибровочная теория на дуальном торе. Калибровочные теории Хитчина появляются при различных обобщениях или вырождениях торической геометрии.

Покажем, как выглядят бранные конфигурации, ответственные за 4-мерные $N=2$ суперсимметричные калибровочные теории с присоединенной или фундаментальной материей. Теории поля определены на мировой поверхности $N_{c}$ параллельных бран, погруженных в дополнительные размерности. Для получения теории в четырех измерениях необходимо иметь четыре некомпактных измерения, а координаты в других измерениях отождествляются с вакуумными значениями скалярных компонент присоединенных гипермультиплетов теории на мировой поверхности браны.

Удобной исходной точкой рассмотрения является мягко нарушенная $N=4$ теория, в которой в терминах $N=1$ теории имеются три гипермультиплета в присоединенном представлении, комплексные скалярные компоненты которых параметризуют вложения бран в шесть измерений. Потенциальная энергия скалярных полей содержит вклад от кинетического члена

$$
V=\frac{1}{g^{2}} \operatorname{Tr} \sum_{i=1,2,3}\left[\phi_{i}, \phi_{i}^{+}\right]^{2},
$$

а также вклад от суперпотенциала

$$
W=\operatorname{Tr} \Phi_{1}\left[\Phi_{2}, \Phi_{3}\right]+M^{2} \operatorname{Tr}\left(\Phi_{2}^{2}+\Phi_{3}^{2}\right),
$$

где $\Phi_{i}-N=1$ поля в присоединенном представлении, а $M$ - масса гипермультиплета. Минимум потенциальной энергии определяет вакуумную конфигурацию, в которой три скалярных поля удовлетворяют нетривиальным коммутационным соотношениям. В пределе большой массы флуктуации полей, ортогональные алгебраической поверхности в двумерном комплексном пространстве

$$
\operatorname{Tr}\left(\Phi_{2}^{2}+\Phi_{3}^{2}\right)=\text { const }
$$

оказываются замороженными.

Сушествуют два эквивалентных подхода к построению $N=2$ теорий в рамках теории струн, если начать с теории в десяти измерениях. В первом из них рассматривается компактное трехмерное комплексное многообразие Калаби-Яу (КЯ) вблизи сингулярности, обеспечивающей подходящую калибровочную группу [40, 47]. В другом 
подходе, предложенном в работе [42], рассматривается теория поля на мировой поверхности 4-бран, расположенных между двумя солитонными, заряженными в секторе Невё-Шварца (НШ), 5-бранами

$$
\left\{\begin{array}{c|cccccccccc} 
& 0 & 1 & 2 & 3 & 4 & 5 & 6 & 7 & 8 & 9 \\
\text { NS5 } & + & + & + & + & + & + & - & - & - & - \\
\text { D4 } & + & + & + & + & - & - & + & - & - & - \\
\text { D6 } & + & + & + & + & - & - & - & + & + & +
\end{array},\right.
$$

где плюс обозначает направление, вдоль которого брана протяженна, а минус - трансверсальную размерность. Так как 4-браны конечны вдоль направления $x^{6}$, соответствующая теория поля оказывается 4 -мерной $N=2$ калибровочной теорией. В картине IIВ (связанной с многообразием КЯ) исходная суперсимметрия (32 действительных суперзаряда) нарушена до $N=2$ в $d=4$ (восемь действительных суперзарядов) геометрией многообразия КЯ. Браны, на которых определена теория поля, намотаны на сингулярные циклы и дополнительно нарушают суперсимметрию вдвое. В картине IIA пространство предполагается плоским и суперсимметрия нарушается подходяшей конфигурацией пересекающихся бран. Последняя картина может быть поднята в 11-мерную М-теорию, которая в низкоэнергетическом секторе сводится к 11-мерной супергравитации.

5.2. Степени свободы многочастичных систем и браны. Определим степени свободы для 4-мерной $S U(n)$-теории без дополнительной материи в картине IIB / F [100] Подобная теория формулируется на мировой поверхности 7-бран, намотанных на эллиптически расслоенное многообразие К3 [47]. Эллиптический слой расслоения имеет комплексную структуру, определяемую затравочной константой связи калибровочной теории, поэтому затравочная спектральная кривая, присутствующая в модели Калоджеро, возникает в построении с самого начала.

Для идентификации степеней свободы необходимо определить на спектральной кривой голоморфную 1-форму $\Phi$ и антиголоморфный векторный потенциал $\bar{A}$. Голоморфная форма может быть найдена, если учесть, что тор вкладывается в многообразие К3 как суперсимметричный цикл и вложение 7-бран в многообразие К3 в этом случае описывается в терминах 1-формы [49], которая в итоге оказывается оператором Лакса для многочастичной системы. Антиголоморфное векторное поле $\bar{A}$, диагональные элементы которого становятся координатами частиц в многочастичной системе, задается $N_{c}$ бранами с калибровочным полем на мировой поверхности, намотанными на спектральную кривую. Последняя составляющая картины - отмеченная точка на спектральной кривой с нетривиальной монодромией - вводится с помощью дополнительных фоновых бран, локализованных на спектральной кривой. Подобные фоновые браны играют роль регуляторов в теории поля. Подчеркнем, что если рассмотрение проводится в рамках F-теории, то роль 11-го и 12-го измерений сводится к геометрическому введению в теорию затравочной константы связи.

Так как введение фоновых бран не меняет интерпретацию степеней свободы, полезно обсудить простейший случай свободной многочастичной системы. Наиболее иллюстративен случай тригонометрической системы Рюсенара, для которого геометрия бран 
находится во взаимнооднозначном соответствии с картиной гамильтоновой редукции, обсуждавшейся в разделе 3 [93]. Напомним, что система возникает из лагранжиана ЧС, определенного на произведении тора на временной интервал. Соотношение для монодромий калибровочного поля по циклам на торе $\mathrm{g}_{A}, \mathrm{~g}_{B}$ имеет вид

$$
\mathrm{g}_{A} \mathrm{~g}_{B} \mathrm{~g}_{A}^{-1} \mathrm{~g}_{B}^{-1}=1
$$

т.е. в свободном случае матрицы могут быть диагонализованы одновременно, причем диагональные элементы матриц определяют координаты и импульсы динамической системы $\mathrm{g}_{A}=\operatorname{diag}\left(e^{\mathrm{i} x_{i}}\right), \mathrm{g}_{B}=\operatorname{diag}\left(e^{\mathrm{i} p_{i}}\right)$. Заметим, что с точки зрения F-теории тор в $11-\mathrm{M}$ и 12-м измерениях вырожден в цилиндр.

Сравним геометрическое описание, следуюшее из лагранжиана ЧС, и бранную конфигурацию. Для описания 5 -мерной теории сушественны две координаты $x^{5}, x^{6}$, причем характерный масштаб по координате $x^{5}$ оказывается равным $R_{5}^{-1}$, а по координате $x^{6}$ равным $1 / g^{2}$ [101]. Мы будем предполагать, что обе координаты компактифицированы на окружности соответствуюших радиусов.

Покажем, что торы в модели Рюсенара связаны с торами в бранной конфигурации преобразованием $T$-дуальности по обеим переменным. Таким образом, в модели Рюсенара радиусы торов равны $\alpha^{\prime} R_{5}, \alpha^{\prime} g^{2}$, а при преобразовании $T$-дуальности собственные числа матрищы монодромии вдоль окружности превращаются в положения бран на дуальной окружности. Точнее, мы имеем $n$ 5-бран в теории IIB, протяженных вдоль направления $x^{6}$ и локализованных в точках $p_{i}$ вдоль $x^{5}$. Координаты в динамической системе задаются $n$ 1-бранами, локализованными в точках $x_{i}$ вдоль $x^{6}$. Суперсимметричная теория поля определена на координатах $\left(x^{0}, x^{1}, x^{2}, x^{3}, x^{4}, x^{6}\right)$, причем координаты 5-бран вдоль $x^{5}$ определяют вакуумные средние вещественного скалярного поля.

Для “включения" взаимодействия в динамическую систему на полевом языке необходимо добавить массивный гипермультиплет в присоединенном представлении, ввести отмеченную точку с нетривиальной монодромией в описании ЧС или ввести дополнительные фоновые браны на языке теории струн.

Новое соотношение на матрицы монодромии выглядит следуюшим образом:

$$
\mathrm{g}_{A} \mathrm{~g}_{B} \mathrm{~g}_{A}^{-1} \mathrm{~g}_{B}^{-1}=\mathrm{g}_{c}
$$

где $\mathrm{g}_{c}$ соответствует $C P(n-1)$-орбите в отмеченной точке. После преобразования $T$-дуальности возникает бранная конфигурация с нетривиальным потоком магнитного поля. Цветовая ориентация потоков поля задается структурой матрицы монодромии $\mathrm{g}_{c}$.

Другой подход к описанию 4-мерных теорий основан на построении теории поля на мировой поверхности М5-браны - солитонного состояния в 11-мерной М-теории [42]. Конфигурация М5-браны должна обеспечить в теории поля сушествование восьми действительных суперзарядов, присутствующих в $N=2$ теории. Этого можно достичь, если рассмотреть в качестве мировой поверхности М5-браны произведение $\mathbb{R}^{4}$-пространства-времени в 4 -мерной теории на компактную поверхность $\Sigma$ фиксированного рода. После компактификации на окружность М5-брана в картине IIA выглядит как набор 4-бран, расположенных между двумя 5-бранами НШ. 
Для получения $S U(n)$-теории необходимо ввести $n$ 4-бран, конечных по одной координате. Координаты бран вдоль направления $x^{4}+\mathrm{i} x^{5}$ в пертурбативном режиме определяют импульсные переменные в многочастичной системе. Кроме того, на поверхности 4-бран имеются $n$ 0-бран, по одной на каждой 4-бране, которые упорядочены вдоль координаты $x^{6}$. В низкоэнергетическом пределе теории поля координаты 0-бран определены следующим образом:

$$
x_{i}=i \Delta+\phi_{i}
$$

где $\Delta$ - постоянная, определяюшая их положения равновесия, $\phi_{i}$ соответствуют малым флуктуациям и совпадают с переменными в системе Тоды. Заметим, что переменные $x_{i}$ могут рассматриваться в качестве координат в системе Калоджеро, в которой любая упорядоченность координат отсутствует. Из сравнения с величинами в теории поля немедленно получаем $n \Delta=1 / g^{2}(M)$, что хорошо согласуется с предложенной упорядоченностью 0-бран вдоль направления $x^{6}$.

Качественная интерпретация локального оператора Лакса в матрицах ранга 2 выглядит следующим образом. Элемент $L_{11}$ соответствует тому, что 0-брана лежит на 4-бране, поэтому им может быть приписана одинаковая координата вдоль направления $x^{4}+\mathrm{i} x^{5}$. Так как операторы Лакса ответственны за фермионные нулевые моды в топологически нетривиальном фоновом поле, то размер матрицы Лакса говорит о сушествовании двумерного пространства нулевых мод. Элементы $L_{12}, L_{21}$ фиксируют матричный элемент спектрального параметра между фермионными нулевыми модами, локализованными на соседних 0-бранах.

Отметим еще раз, что в любой бранной конфигурации обязательно присутствуют три элемента. Во-первых, вводятся браны, на мировой поверхности которых реализуется теория поля: $n$ 7-бран в картине IIB/ $F$ или М5-брана в картине IIA/ $M$. Во-вторых, необходимо ввести браны, задающие координаты в динамической системе: с точки зрения теории поля они являются инстантонами нулевого радиуса [102]. И, в-третьих, введение регуляторов в теории поля отвечает введению вильсоновской линии в картине IIB $/ F$ или "магнитных потоков" в описании IIA/ $M$.

5.3. Браны в 5-мерной теории. Покажем, что при обсуждении 5-мерных калибровочных теорий роль бранной конфигурации становится еще более прозрачной. Оказывается, что в 5-мерной теории бранная диаграмма фиксирует структуру спектральной кривой интегрируемой системы. Ниже мы рассмотрим бранную конфигурацию в картине IIB, которая связана с данными торического многообразия, описываюшего компактификацию М-теории [40, 47, 63].

В качестве компактного многообразия мы выбираем многообразие КЯ вблизи особых точек, например вблизи $A_{n-1}$ ALE-пространств, расслоенных над базой, являющейся набором сфер, радиус которых задает константы связи $V \propto 1 / g^{2}$ в соответствующем $S U(n)$-факторе. Кэлеровы модули, задаваемые набором $P^{1}$ при раздутии ALE-сингулярностей, определяют координаты на кулоновской ветви пространства модулей. Так как многообразие может быть представлено как гиперповерхность в голоморфном многообразии, то оно может быть задано набором кэлеровых конусов или торическим многогранником $\boldsymbol{\Delta}$. 
Например, если рассмотреть $N=(2,2) \quad \sigma$-модели [103], получим $U(1)$-вектор зарядов материи

$$
\mathbf{Q}^{a}=\left(q_{1}^{a}, \ldots, q_{k}^{a}\right)
$$

и однородные координаты, связанные соотношениями

$$
\sum_{J} Q_{J}^{a} \nu_{J}=0
$$

между векторами $\nu_{J} \in \mathbb{N}=\mathbb{Z}^{d}$. Вершины $\nu_{J}$ в решетке $\mathbb{N}$ образуют торический многогранник $\boldsymbol{\Delta}=\left\{\nu_{J}\right\}$. Для того чтобы многообразие было гладким, многогранник $\boldsymbol{\Delta}$ должен быть замкнутым. Таким образом, сформулировав структуру многообразия в терминах торических диаграмм, можно перейти к решению модели.

Как известно [40, 47], кэлеровы модули исходного многообразия КЯ становятся координатами кулоновской ветви пространства модулей низкоэнергетической калибровочной теории. После преобразования зеркальной симметрии кэлеровы модули превращаются в модули комплексной структуры зеркального многообразия, которое оказывается римановой поверхностью. Риманова поверхность является не чем иным, как спектральной кривой соответствующей интегрируемой системы. В 5-мерном случае интегрируемая система является анизотропной неоднородной $X X Z$-спиновой цепочкой, а в рассмотренном в предыдущем разделе примере становится релятивистской системой Тоды [62].

В терминах торических диаграмм зеркальное многообразие задается торической диаграммой на дуальной решетке [7], причем каждый дуальный кэлеров конус $\Sigma$ задается векторами $\mu \in \widetilde{\Sigma}$. В терминах векторов зарядов зеркальное многообразие имеет вид

$$
\prod_{J} y_{J}^{q_{J}^{a}}=1
$$

Именно это выражение и определяет спектральную кривую интегрируемой системы.

Перейдем к описанию 5-мерных теорий в терминах бранных диаграмм. Удобно получать бранную конфигурацию с помошью преобразования $T$-дуальности вдоль направления $x^{4}$ из картины типа IIA [42]. Таким образом, мы получаем картину типа IIB с бранной конфигурацией [101]

$$
\left\{\begin{array}{c|cccccccccc} 
& 0 & 1 & 2 & 3 & 4 & 5 & 6 & 7 & 8 & 9 \\
\text { NS5 } & + & + & + & + & + & + & - & - & - & - \\
\text { D5 } & + & + & + & + & + & - & + & - & - & -
\end{array},\right.
$$

где теория поля реализуется на мировой поверхности D5-браны. Голоморфное решение уравнения Лапласа в 4-мерном пространстве в пяти измерениях имеет вид

$$
x^{6}=\frac{1}{2}\left|x^{5}\right|=\frac{x^{5}}{2} \operatorname{sign} x^{5} .
$$

Если потребовать нарушение суперсимметрии только в 4 раза, то возникнет ограничение на структуру пересечения бран. В частности, при пересечении 5-браны с зарядом $n$ 
и 5-браны НШ с зарядом $k$ они образуют связанное состояние $(k, n)$, распространяюшееся под углом $\phi=\operatorname{arctg}(k / n)$ на плоскости $\left(x^{5}, x^{6}\right)[101]$.

В терминах бран вводятся все переменные в теорию поля, и естественно разделить все модули на два класса: влияющие и не влияющие на асимптотику бран. Параметры, меняюшие асимптотику, становятся константами связи и затравочными массами в 5-мерной теории, а переменные, не меняющие асимптотику, становятся динамическими модулями. Уместно пояснить, как торические диаграммы связаны с подходом, предложенным в работах $[40,47]$. Каждая грань торического многогранника $\boldsymbol{\Delta}$ эквивалентна 1-циклу на римановой поверхности $E$. Периоды 1 -циклов в компактной части $E$ (т.е. внутренние грани в $\boldsymbol{\Delta}$ ) определяют динамические модули, а циклы по некомпактной части определяют затравочные параметры модели (т.е. внешние грани торической диаграммы $\boldsymbol{\Delta}$ ) [7]. Подобная интерпретация согласована с тем фактом, что число внешних граней $\boldsymbol{\Delta}$ в конкретной теории поля равно чис лу бесконечных 5-бран в соответствующей бранной конфигурации. Покажем теперь, что бранная конфигурация и торическая диаграмма в 5-мерном случае тесно связаны друг с другом. С этой целью удобно приписать бранной диаграмме кинематику свободной частицы в двумерном пространстве, тогда условие сохранения импульса в вершине эквивалентно сохранению заряда при отождествлении $p^{(5,6)}=(k, n)$ с $S L(2, \mathbb{Z})$-зарядом браны. Согласно стандартным правилам вертикальные браны имеют вектор заряда $(1,0)$, а горизонтальные - $(0,1)$. Напомним, что число вертикальных бран НШ определяет число калибровочных групп, а число горизонтальных 5-бран определяет ранг в каждом $S U$-факторе. Например, для описания $S U(n)^{k}$-калибровочной теории необходимо $k+1$ бран НШ и $n 5$-бран, натянутых между каждой парой бран НШ. Соответственно число вертикальных граней в торической диаграмме равно $k+1$, а число горизонтальных граней равно $n$. В общей точке пространства модулей вершины $\boldsymbol{\Delta}$ лежат на двумерной целочисленной решетке. При этом каждому ребру ставится в соответствие целочисленный двумерный вектор $\left(k_{\Delta}, n_{\boldsymbol{\Delta}}\right)$. Ключевым является утверждение о соответствии между бранной диаграммой теории IIВ и торической диаграммой многообразия КЯ при компактификации М-теории к 5-мерной теории поля. Эквивалентность достигается отождествлением двумерного вектора импульса $\left(k_{\boldsymbol{\Delta}}, n_{\boldsymbol{\Delta}}\right)$ с вектором, отвечающим ребру торической диаграммы.

Найденное соответствие позволяет решать любую 5-мерную калибровочную теорию с группой $\prod_{i=1}^{k} S U\left(n_{i}\right)$ и произвольной материей. Торический многогранник $\boldsymbol{\Delta}$ для недеформированной $S U(n)$-калибровочной теории приведен в работе [47]. Добавление массивного кварка эквивалентно разрыву одной внешней линии в торической диаграмме и добавлению одной внутренней. Таким образом, общее число линий на диаграмме увеличивается на одну, это эквивалентно введению одного нового параметра - массы кварка, что полностью согласуется с бранной диаграммой.

Другой способ введения фундаментальной материи состоит в добавлении новых полубесконечных бран. На языке бран это может быть сделано четырьмя способами в терминах исходных полубесконечных бран. Столько же неэквивалентных возможностей имеется при разрыве внешних ребер торических диаграмм на две части. Для группы $\prod_{i=1}^{k} S U\left(n_{i}\right)$ необходимо брать расслоение $n_{i}$ сфер над каждой из $k$ сфер на базе. 
Обшее число внешних ребер в торической диаграмме равно $2(k+1)$ и совпадает с числом полубесконечных бран в подходяшей конфигурации. Число конечных бран равно $\sum_{i=1}^{k}\left(3 n_{i}-1\right)-1$ в согласии с числом внутренних ребер в торической диаграмме.

5.4. Уравнения движения в многочастичных системах и браны. Следующим шагом в обосновании интерпретации коллективных координат бран как степеней свободы интегрируемой системы является выяснение роли классических уравнений движения в бранной конфигурации [100]. С этой целью мы свяжем бранную конфигурацию с пространством модулей монополей и отождествим уравнения движения с уравнениями Нама, решения которых определяют характеристики пространства модулей.

В качестве степеней свободы, отвечающих монополям, можно рассматривать 0-браны, локализованные на 4-бранах. 0-браны могут быть связаны с другими 4-бранами открытыми струнами. Следуя [104] теорию на мировой поверхности струн можно получить размерной редукцией из 10-мерной теории ЯМ. Более того, имеется однозначное соответствие между уравнением, выделяюшим суперсимметричное вакуумное состояние в $\sigma$-модели, и уравнением Нама для монополей. Последним необходимым шагом в получении уравнений движения в системе Тоды является учет связи между спектральной кривой в системе Тоды и спектральной кривой для $N_{c}$-циклической монопольной конфигурации, следующей из уравнения Нама [105]. Спектральная кривая для циклической конфигурации может быть получена из кривой обшего положения

$$
\eta^{n}+\eta^{n-1} a_{1}(\kappa)+\cdots+a_{n}(\kappa)=0
$$

в предположении, что центр масс системы монополей зафиксирован в начале координат, общая $U(1)$-фаза положена равной единице и наложено условие симметрии относительно циклической группы порядка $N_{c}$.

Указанная выше цепочка аргументов позволяет связать оператор Лакса для системы Тоды с известными в теории монополей [106] матрицами Нама

$$
\begin{gathered}
T=T_{1}+\mathrm{i} T_{2}-2 \mathrm{i} T_{3} \rho+\left(T_{1}-\mathrm{i} T_{2}\right) \rho^{2} \\
T_{1}=\frac{\mathrm{i}}{2} \sum_{j=1} q_{j}\left(E_{+j}+E_{-j}\right), \quad T_{2}=-\sum_{j=1} q_{j}\left(E_{+j}-E_{-j}\right) \\
T_{3}=\frac{\mathrm{i}}{2} \sum_{j=1} p_{j} H_{j}
\end{gathered}
$$

где $E$ и $H$ - стандартные генераторы группы $S U(N), p_{j}, q_{j}$ представляют фазовое пространство в системе Тоды, а $\rho$ - координата на твисторной поверхности $C P^{1}$. Точка на $C P^{1}$ определяет комплексную структуру на пространстве модулей монополей. Окончательно уравнения движения в системе Тоды отождествляются с уравнениями Нама, записанными в форме

$$
\frac{d T}{d t}=[T, A]
$$

при фиксированном А. 
Для дальнейшего описания геометрической картины полезно рассмотреть фермионы во внешнем поле, т.е. оператор Дирака во внешнем монопольном поле

$$
(\sigma D+\phi-t) \Psi=0
$$

Уравнение имеет $k$ линейно независимых решений для магнитного заряда $k$. Таким образом, именно переменная $t$ становится временной переменной в системе Тоды, и одновременно она является пространственной координатой в бранной интерпретации. Матрицы Нама в терминах фермионных нулевых мод выглядят следуюшим образом:

$$
T_{j}=\int x_{j} \Psi^{+} \Psi d^{3} x \quad(j=1,2,3), \quad T_{4}=\mathrm{i} \int \Psi^{+} \frac{d \Psi}{d t} d^{3} x .
$$

Оба выражения имеют структуру связности Берри, если рассматривать импульсное пространство в качестве пространства параметров. Существует и обратная конструкция, позволяющая получить поля мультимонопольной конфигурации, начиная со вспомогательной задачи для фермионов в поле, задаваемой матрицами Нама

$$
\begin{gathered}
\left(\mathrm{i} \frac{d}{d t}+\mathrm{i} \sigma x+\sigma T^{+}\right) v=0, \\
A_{j}=\int v^{+} \partial_{j} v d t, \quad \phi=\int t v^{+} v d t,
\end{gathered}
$$

где пределы интегрирования определяются асимптотическими значениями поля Хиггса. При этом фермионы, рассмотренные в точке $x=0$, отождествляются с фермионами в спектральной задаче для системы Тоды.

Рассмотрим обобшение конструкции Нама на случай $S U(p)$ [104]. Зафиксируем асимптотику $n$ компонент поля Хиггса равной $\mu_{i}, i=1, \ldots, n, \sum_{i} \mu_{i}=0$, после чего область определения уравнения Нама разбивается на $n-1$ интервалов $\left(\mu_{1}, \mu_{2}\right), \ldots,\left(\mu_{n-1}, \mu_{n}\right)$. На каждом $a$-м интервале определены три $\left(p_{k} \times p_{k}\right)$-матрицы Нама $\mathcal{T}_{i, a}(t)$ :

$$
\frac{d \mathcal{T}_{i, a}}{d t}=\frac{1}{2} \epsilon_{i j k}\left[\mathcal{T}_{j, a}, \mathcal{T}_{k, a}\right]
$$

Таким образом, монополь в случае $S U(n)$ определяется набором топологических зарядов $p_{1}, \ldots, p_{n-1}$.

Соответствие между решениями уравнений Нама и спектральными кривыми интегрируемых систем обобшается на случай $S U(p)$ [104], для которого имеется связь с магнетиками высших спинов [93]. Решение уравнений Нама задается кривой, являюшейся произведением $n-1$ компонент, каждая из которых является спектральной кривой, отвечающей группе $S U(2)$, с магнитным зарядом $p_{a}$. Таким образом, полная спектральная кривая является специальным случаем кривой для магнетика спина $p=\sum_{a} p_{a}$ на $n$ узлах. Конфигурация в магнетике выделена тем, что происходит кластеризация узлов на пары, каждая из которых описывает $S L\left(p_{a}\right)$-магнетик. Между парами узлов наложены дополнительные условия согласования. 
Выделенность конфигурации спиновых цепочек имеет ясное объяснение на языке бран. Монопольное пространство модулей строится из конфигурации 1-бран и 3-бран [104]. Кластеризация на языке бран означает, что 1-браны могут оканчиваться только на соседних 3-бранах. Отождествление спиновых цепочек с бранной конфигурацией достигается, если считать, что 3-браны задают узлы спиновой решетки, а 1-браны, натянутые меж ду ними, определяют группу $S L(p)$, где $p$ - полное число 1-бран. Заметим, что использованная процедура твистования цепочки оказывается эквивалентна добавлению полубесконечных 1-бран к самой левой и самой правой 3-бранам.

5.5. Браны и два представления Лакса. Рассмотрим два представления Лакса, известных в ряде интегрируемых систем, и покажем, что их существование имеет ясную интерпретацию в терминах бранных диаграмм [93]. Простейшим примером может служить система Тоды, допускающая представления Лакса в терминах $(2 \times 2)-$ и $(n \times$ $n$ )-матриц. В первом случае система формулируется в терминах трансфер-матрицы, определенной на $n$ узлах, а во втором случае понятие трансфер-матрицы ввести невозможно. Оказьвается, что разные представления Лакса связаны между собой врашением бранных конфигураций.

Для системы Тоды имеем

$$
L_{\text {Toda }}=\left(\begin{array}{cc}
\lambda+p & e^{q} \\
-e^{-q} & 0
\end{array}\right)
$$

Система может быть переформулирована в терминах $(n \times n)$-матриц следуюшим образом. Вводится двухкомпонентная функция Бейкера-Ахиезера (БА $\Psi_{n}=\left(\begin{array}{c}\psi_{n} \\ \chi_{n}\end{array}\right)$, в терминах которой линейная задача имеет вид

$$
\psi_{n+1}-p_{n} \psi_{n}+e^{q_{n}-q_{n-1}} \psi_{n-1}=\lambda \psi_{n}, \quad \chi_{n}=-e^{q_{n-1}} \psi_{n-1}
$$

и согласно периодическим граничным условиям сводится к линейной задаче

$$
\mathcal{L}(w) \Phi=\lambda \Phi
$$

для $(n \times n)$-оператора Лакса

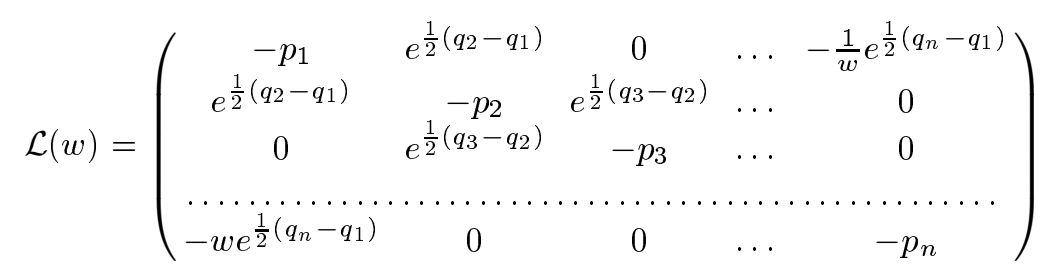

с $n$-компонентной функцией БА $\Phi=\left\{e^{-q_{n} / 2} \psi_{n}\right\}$. Уравнение задает спектральную кривую

$$
\operatorname{det}_{n \times n}(\mathcal{L}(w)-\lambda)=0
$$

с правильными свойствами.

С точки зрения бранной конфигурации два представления Лакса отвечают выбору параметризации мировой поверхности $\Sigma$ 5-браны. Голоморфные координаты $\lambda=x^{4}+$ 
i $x^{5}$ и $s=x^{6}+\mathrm{i} x^{10}$ могут быть выбраны в качестве спектрального параметра. В $(2 \times$ $2)$-представлении в качестве спектрального параметра выбирается $w=\exp (-s / R)$, а другой выбор отвечает $(n \times n)$-представлению.

Геометрически обсуждаемое явление можно связать с симметрией между базой и слоем в трехмерном многообразии КЯ [47]. В $(2 \times 2)$-представлении переменная $w$ является координатой на базе с сингулярностью типа $A_{m}$ и учитывает число факторов в полной калибровочной группе, а $\lambda$ - координата в слое. Коэффициенты перед членами $w^{i}$ являются полиномами степени $n$, а полная калибровочная группа $-S U(n)^{m}$ с дополнительными фундаментальными гипермультиплетами на краях диаграммы. Дуальность $S U(n)^{m} \leftrightarrow S U(m+1)^{n-1}$, предложенная в работе [47], является не чем иным, как переходом между двумя представлениями Лакса. При действии симметрии происходит изменение роли координат на многограннике Ньютона, описывающем многообразие.

Применим приведенную выше аргументацию к описанию фундаментальной материи в 4-мерной калибровочной теории. Имеются две реализации материи на языке бран в терминах полубесконечных D4- и D6-бран. Покажем, что в терминах интегрируемых систем первая реализация отвечает твистованной цепочке Тоды, а вторая - $X X X$ магнетику.

Ключевым фактом является наблюдение [39], что при пересечении двух бран происходит рождение новых бран. В простейшем случае конфигурации

$$
\left\{\begin{array}{c|cccccccccc} 
& 0 & 1 & 2 & 3 & 4 & 5 & 6 & 7 & 8 & 9 \\
\mathrm{NS} 5 & + & + & + & + & + & + & - & - & - & - \\
\mathrm{D} 3 & + & + & + & - & - & - & + & - & - & - \\
\mathrm{D} 5 & + & + & + & - & - & - & - & + & + & +
\end{array}\right.
$$

при прохож дении D5-браны через NS5-брану рождается новая D3-брана, что после преобразования $T$-дуальности эквивалентно рождению D4-бран при движении D6-бран.

Кривая в теории с фундаментальной материей может быть получена из $(n \times n)$-представления деформацией оператора Лакса и добавлением в первый и последний столбцы ненулевых элементов [107], что соответствует добавлению полубесконечных бран к краям конфигурации. В $(2 \times 2)$-представлении фундаментальной материи отвечают D6-браны, локализованные в точках, определяемых массами, что согласуется со связью масс и неоднородностей в спиновой цепочке. Два представления могут быть связаны, если использовать явление рождения новых бран при пересечении с NS5-браной.

5.6. Динамика Уизема в терминах бран. Напомним, что в суперсимметричной калибровочной теории мы встретились с двумя интегрируемыми системами разной природы. Интерпретация системы Тоды в терминах бран была предложена выше, поэтому мы переходим к обсуждению динамической системы уиземовского типа. В качестве исходной бранной конфигурации удобно выбрать F-теорию, определенную на эллиптически расслоенном многообразии К 3 , что эквивалентно ориентифолду в теории IIB.

Согласно [108] мы можем рассматривать $N=2$ 4-мерную теорию как теорию на мировой поверхности 3-бран в фоне расшепленных ориентифолдов, расположенных в точках $\pm \Lambda$ на комплексной плоскости $u=\operatorname{Tr} \phi^{2}$ для калибровочной группы $S U(2)$. Мы 
предполагаем, что все массы фундаментальной материи велики, поэтому мы можем работать с недеформированной теорией ЯМ.

Рассмотрим динамику 3-бран по координатам, ортогональным фоновым 7-бранам. Ниже мы ограничимся случаем калибровочной группы $S U(2)$, однако возникающая динамика имеет ясную интерпретацию, поэтому обобщение на группы более высокого ранга прямолинейно. Напомним, что для групшы $S U(2)$ динамика Уизема задается решением Гуревича-Питаевского задачи о модулящии однозонного решения уравнения Кд $\Phi$. В терминах бран решение формулируется следуюшим образом.

В начальный момент времени 3-браны совпадают с одной из 7-бран, в процессе эволюции происходит "рассеяние" 3-бран друг на друге, после чего они поглошаются другой парой 7-бран. Таким образом, точная метрика на пространстве модулей задается динамикой обмена 3-бранами, что обобшает картину обмена замкнутыми струнами, предложенную в работе [109].

Удобно рассматривать спектральную кривую в следующей параметризации:

$$
y^{2}=\left(x^{2}-\Lambda^{2}\right)(x-u)
$$

Координата $u$ на пространстве модулей определяет положения двух 3-бран, находяшихся в точках $\pm \sqrt{u}$ на плоскости $\phi$, а остальные точки ветвления определяют положения фоновых бран. Точка ветвления, отвечаюшая динамическим бранам, движется согласно уравнениям Уизема для однозонного решения от $u=\Lambda$ до $u=-\Lambda$. Явное решение имеет вид

$$
\eta(x, t)=2 d n^{2}\left[\frac{1}{\sqrt{6}}\left(x-\frac{1+s^{2}}{3} t, s\right)\right]-\left(1-s^{2}\right),
$$

где

$$
\frac{1+s^{2}}{3}-\frac{2 s^{2}\left(1-s^{2}\right) K(s)}{3\left(E(s)-\left(1-s^{2}\right) K(s)\right)}=\frac{x}{t},
$$

$K(s)$ и $E(s)$ - эллиптические модули кривой, а $s^{2}=(u+\Lambda) / 2 \Lambda$. В автомодельной переменной $\theta=x / t$ левая фоновая брана находится при $\theta=-1$, а правая - при $\theta=2 / 3$. Квазиклассическая $\tau$-функция решения определяет препотенциал эффективной $S U(2)$-теории согласно формуле $\mathcal{F}=\log \tau_{\mathrm{qcl}}$.

Подчеркнем аналогию между рассмотренной выше динамикой бран и рассеянием монополей при низких энергиях. Действительно, сушествует геодезическая траектория, движение вдоль которой приводит к преврашению монополей в дионы [110]. Рассмотренная выше эволюция бран эквивалентна этому процессу в следуюшем смысле. В начальный момент эволюции, когда браны находятся в точках $\pm \sqrt{\Lambda}$, согласно точному выражению для БПС-спектра в теории имеются безмассовые монополи. На плоскости $\phi$ браны расположены в точках $\pm \phi$, а фоновые браны - в точках $\pm \sqrt{\Lambda}, \pm \mathrm{i} \sqrt{\Lambda}$. После рассеяния под прямым углом в точке $u=0$ браны приобретают электрический заряд: в конце эволюции, когда динамические браны оказываются в точке $u=-\Lambda$, в теории имеются безмассовые дионы, но, с другой стороны, дионы могут рассматриваться как $(1,1)$-струны между динамическими и фоновыми бранами [111], т.е. динамические браны обязательно должны приобретать электрический заряд. 


\section{6. ЗАКЛЮЧЕНИЕ}

Мы постарались прояснить роль и интерпретацию классической интегрируемости в контексте вакуумного сектора суперсимметричных калибровочных теорий. Вакуумный сектор описывается в терминах пространств модулей, которые являются фазовыми пространствами пары интегрируемых систем. Системы Калоджеро-Тоды определяют динамику на пространстве модулей инстантонов, которая может быть сформулирована в терминах топологических калибровочных теорий в “импульсном” пространстве. Динамические системы уиземовского типа имеют интерпретацию ренормгрупповых потоков в теории и формулируются на кулоновской ветви пространства модулей. Обе интегрируемые системы описываются как динамика коллективных координат бран в разных направлениях.

Несмотря на то что справедливость и эффективность подхода не вызывают сомнений, его обоснование из первых принципов отсутствует. Наиболее важной задачей представляется суммирование бесконечного инстантонного ряда в конечное число D0-бран, координаты которых задают фазовое пространство интегрируемой системы. В последнее время предпринимаются попытки переформулировать задачу в других, более формальных терминах, что возможно позволит продвинуться на пути к ее решению с помошью современных математических методов [112]. Не менее важным с концептуальной точки зрения представляется выяснение роли квантования интегрируемой системы. Роль соответствуюшей "постоянной Планка" пока совершенно неясна.

Сейчас уже представляется очевидным, что браны чрезвычайно важны в непертурбативной области и играют роль, аналогичную роли диаграмм Фейнмана в теории возмущений. Мы показали, что в простейших случаях интегрируемость может быть описана в терминах динамики бран, однако исчерпывающая формулировка их связи пока отсутствует, и несомненно требуется дальнейшая работа в этом направлении.

Благодарности. Автор благодарен С. Гукову, А. Маршакову, А. Миронову, А. Морозову, И. Кричеверу, Н. Некрасову, В. Рубцову, В. Фоку за участие в совместных работах, на которых основан настоящий обзор, а также А. Герасимову, А. Забродину, А. Лосеву, М. Ольшанецкому, А. Рослому, К. Селиванову за полезные обсуждения. Работа была частично поддержана грантами РФФИ № 97-02-16131 и INTAS-97-0103.

\section{Список литературы}

[1] N. Seiberg, E. Witten. Nucl. Phys. B. 1994. V. 426. P. 19.

[2] N. Seiberg, E. Witten. Nucl. Phys. B. 1994. V. 431. P. 484.

[3] Б. А.Дубровин, И. М. Кричевер, С. П. Новиков. Интегрируемые системы-І. В сб.: Итоги науки и техники. Сер. Современные проблемы математики. Фундаментальные направления. Т. 4. Динамические системы-4. Ред. Р. В. Гамкрелидзе. М.: ВИНИТИ, 1985. С. 179; Б. А. Дубровин. УМН. 1981. Т. 36. № 2. С. 11.

[4] B. Dubrovin, S. Novikov. Sov. Sci. Rev. Sect. C: Math. Phys. Rev. 1993. V. 9. № 4. P. 1; I. Krichever. Sov. Sci. Rev. Sect. C: Math. Phys. Rev. 1992. V. 9. № 2. P. 1.

[5] A. Bilal. Duality in $N=2$ SUSY $S U(2)$ YM theory. A pedagogical introduction to the work of Seiberg and Witten. hep-th/96010010; W. Lerche. Introduction to Seiberg-Witten theory and its stringy origin. hep-th/9611190. 
[6] A. Giveon, D. Kutasov. Brane dynamics and gauge theories. hep-th/9802031; J. Schwarz. Power of M-theory. hep-th/9601077; W. Taylor. Lectures on D-branes, gauge theory and $\mathrm{M}$ (atrices). hep-th/9801182.

[7] A. Klemm. On the geometry behind $N=2$ supersymmetric effective actions on four dimensions. hep-th/9711142.

[8] А. Ю. Морозов. УФН. 1992. Т. 162. С. 84; 1994. Т. 164. С. 3.

[9] A. Marshakov. Seiberg-Witten theory and integrable systems. Singapore: World Scientific, 1999.

[10] M. Shifman. Phys. Rep. 1991. V. 209. P. 343.

[11] A. Migdal, M. Shifman. Phys. Lett. B. 1982. V. 114. P. 445.

[12] G. Veneziano, S. Yankielowicz. Phys. Lett. B. 1982. V. 113. P. 231.

[13] C. Vafa, S. Cecotti. Commun. Math. Phys. 1993. V. 158. P. 569.

[14] M. Fukuma, H. Kawai, R. Nakayama. Commun. Math. Phys. 1992. V. 143. P. 371.

[15] R. Dijgraaf, E. Verlinde, H. Verlinde. Nucl. Phys. B. 1991. V. 352 . P. 59.

[16] M. Douglas. Phys. Lett. B. 1990. V. 238. P. 176.

[17] M. Atiyah, V. Drinfeld, N. Hitchin, Yu. Manin. Phys. Lett. A. 1978. V. 65. P. 17.

[18] M. Douglas. Gauge fields and D-branes. hep-th/9604198.

[19] E. Witten. Nucl. Phys. B. 1990. V. 340. P. 281.

[20] E. Witten. Surv. Diff. Geom. 1991. V. 1. P. 243.

[21] М. Л. Концевич. Функц. анализ и его прилож. 1991. Т. 25. С. 50.

[22] E. Witten. Commun. Math. Phys. 1988. V. 117. P. 353.

[23] S. Donaldson. Duke Math. J. 1987. V. 54. P. 231.

[24] V. Novikov, M. Shifman, A. Vainshtein, V. Zakharov. Nucl. Phys. B. 1983. V. 229. P. 381.

[25] V. Novikov, M. Shifman, A. Vainshtein, V. Zakharov. Nucl. Phys. B. 1983. V. 229. P. 407.

[26] A. Marshakov, A. Mironov, A. Morozov. Phys. Lett. B. 1996. V. 389. P. 43.

[27] M. Shifman, A. Vainshtein. Nucl. Phys. B. 1991. V. 359. P. 571.

[28] N. Seiberg. Power of holomorphy - exact results in $4 d$ SUSY field theories. hep-th/9408013.

[29] C. Montonen, K. Olive. Phys. Lett. B. 1977. V. 78. P. 117.

[30] A. Klemm, W. Lerche, S. Theisen, S. Yankielowicz. Phys. Lett. B. 1995. V. 344. P. 169.

[31] P. Argyres, A. Faraggi. Phys. Rev. Lett. 1995. V. 73. P. 3931.

[32] K. Olive, E. Witten. Phys. Lett. B. 1979. V. 78. P. 97.

[33] J. Polchinski. Phys. Rev. Lett. 1995. V. 75. P. 4724.

[34] J. Polchinski. TASI - lectures on D-branes. hep-th/9611050.

[35] C. Hall, P. Townsend. Nucl. Phys. B. 1995. V. 438. P. 109.

[36] P. Townsend. P-brane democracy. hep-th/9507048.

[37] E. Witten. Nucl. Phys. B. 1996. V. 430. P. 335.

[38] S. Kachru, C. Vafa. Nucl. Phys. B. 1995. V. 450. P. 69.

[39] A. Hanany, E. Witten. Nucl. Phys. B. 1997. V. 492. P. 152.

[40] S. Katz, A. Klemm, C. Vafa. Nucl. Phys. B. 1997. V. 497. P. 173.

[41] H. Ooguri, C. Vafa. Nucl. Phys. B. 1996. V. 463. P. 55.

[42] E. Witten. Solution of $N=2$ supersymmetric theories via M-theory. hep-th/9703166.

[43] P. Townsend. D-branes from M-branes. hep-th/9512062.

[44] P. Townsend. Phys. Lett. B. 1995. V. 350. P. 184.

[45] E. Witten. Branes and the dynamics of QCD. hep-th/9706109.

[46] K. Hori, H. Ooguri, Y. Oz. Strong coupling dynamics of four-dimensional $N=1$ theories from M theory fivebrane. hep-th/9706082.

[47] S. Katz, P. Mayr, C. Vafa. Mirror symmetry and Exact Solution of 4D $N=2$ Gauge Theories - I. hep-th/9706110.

[48] C. Vafa, H. Ooguri. Nucl. Phys. B. 1997. V. 500. P. 62.

[49] M. Bershadsky, V. Sadov, C. Vafa. Nucl. Phys. B. 1996. V. 463. P. 420.

[50] C. Vafa. Nucl. Phys. B. 1996. V. 469. P. 403. 
[51] F. Calogero. J. Math. Phys. 1971. V. 12. P. 419.

[52] J. Moser. Adv. Math. 1975. V. 16. P. 197.

[53] M. Olshanetsky, A. Perelomov. Phys. Rep. 1981. V. 71. P. 313; 1983. V. 94. P. 6.

[54] D. Kazhdan, B. Kostant, S. Sternberg. Commun. Pure Appl. Math. 1978. V. 31. P. 481.

[55] B. Sutherland. Phys. Rev. A. 1972. V. 5. P. 1372.

[56] S. N. M. Ruijsenaars, H. Schneider. Ann. Phys. (NY). 1986. V. 170. P. 370.

[57] S. N. Ruijsenaars. Commun. Math. Phys. 1988. V. 115. P. 127.

[58] A. Gorsky, N. Nekrasov. Nucl. Phys. B. 1994. V. 414. P. 213.

[59] А. С. Горский. ТМФ. 1995. Т. 103. С. 437.

[60] A. Gorsky, N. Nekrasov. Nucl. Phys. B. 1995. V. 436. P. 582.

[61] O. Babelon, D. Bernard. Phys. Lett. B. 1993. V. 317. P. 363.

[62] N. Nekrasov. Five-dimensional gauge theories and relativistic integrable systems. hep-th/9609219.

[63] N. Nekrasov, A. Lowrence. Instanton sums and five-dimensional gauge theories. hep-th/9706025.

[64] A. Gorsky, N. Nekrasov. Elliptic Calogero-Moser system from two-dimensional current algebra. hep-th/9401021.

[65] G. Arutyunov, S. Frolov, P. Medvedev. J. Math. Phys. 1997. V. 38. P. 5682; J. Phys. A. 1997. V. 15. P. 505.

[66] N. Hitchin. Duke Math. J. 1987. V. 54. P. 91.

[67] E. Markman. Compos. Math. 1994. V. 93. P. 255.

[68] V. Inozemtsev. Commun. Math. Phys. 1989. V. 121. P. 629.

[69] A. Gorsky, N. Nekrasov, V. Roubtsov. Hilbert schemes, separated variables and D-branes. hep-th/9901089.

[70] V. Fock, A. Gorsky, N. Nekrasov, V. Roubtsov. Duality in integrable systems and gauge theories. hep-th/9906235.

[71] E. Sklyanin. Separation of variables: New trends. solv-int/9505003.

[72] J. Hurtubise. Duke Math. J. 1996. V. 83. P. 19.

[73] G. Wilson. Invent. Math. 1998. V. 133. P. 1.

[74] H. Nakajima. Lectures on Hilbert Schemes of Points on Surfaces. Providence, RI: AMS, 1999.

[75] E. Frenkel. Affine algebras, Langlads duality and Bethe anzatz. q-alg/9506003.

[76] M. Matone. Phys. Lett. B. 1995. V. 357. P. 342; J. Sonnenschein, S. Theisen, S. Yankielowicz. Phys. Lett. B. 1996. V. 367. P. 145; T. Eguchi, S. K. Yang. Mod. Phys. Lett. A. 1996. V. 11. P. 131; P. Howe, P. West. Nucl. Phys. B. 1997. V. 486. P. 425; E. D'Hoker, I. Krichever, D. H. Phong. Nucl. Phys. B. 1997. V. 494. P. 89.

[77] S. Ruijsenaars. Commun. Math. Phys. 1988. V. 115. P. 127; Finite-dimensional soliton systems. In: Integrable and Super-Integrable Systems. Ed. B. Kupershmidt. Singapore: World Scientific, 1990. P. 165; Publ. RIMS Kyoto Univ. 1994. V. 30. P. 865; 1995. V. 31. P. 247.

[78] G. Braden, A. Marshakov, A. Mironov, A. Morozov. On double-elliptic integrable systems. 1. A duality argument for the case of SU(2). hep-th/9906240.

[79] K. Intrilligator, N. Seiberg. Phys. Lett. B. 1996. V. 387. P. 513.

[80] P. Etingof, A. Varchenko. Traces of intertwiners for quantum groups and difference equations. I. math/9907181.

[81] A. Gorsky, I. Krichever, A. Marshakov, A. Mironov, A. Morozov. Phys. Lett. B. 1995. V. 355. P. 466

[82] E. Martinec, N. Warner. Nucl. Phys. B. 1996. V. 459. P. 97.

[83] T. Nakatsu, K. Takasaki. Mod. Phys. Lett. A. 1996. V. 11. P. 157.

[84] T. Eguchi, S. Yang. Mod. Phys. Lett. A. 1996. V. 11. P. 131.

[85] R. Donagi, E. Witten. Nucl. Phys. B. 1996. V. 460. P. 299.

[86] E. Martinec. Phys. Lett. B. 1996. V. 367. P. 91. 
[87] A. Gorsky, A. Marshakov. Phys. Lett. B. 1996. V. 374. P. 218.

[88] H. Itoyama, A. Morozov. Nucl. Phys. B. 1996. V. 477. P. 855.

[89] H. Itoyama, A. Morozov. Nucl. Phys. B. 1997. V. 491. P. 529.

[90] I. Krichever. The $\tau$-function of the universal Whitham hierarchy, matrix models and topological field theories. hep-th/9205110.

[91] А. В. Гуревич, Л. П. Питаевский. Письма в ЖЭТФ. 1973. Т. 17. С. 268.

[92] A. Gorsky, A. Marshakov, A. Mironov, A. Morozov. Phys. Lett. B. 1996. V. 380. P. 75.

[93] A. Gorsky, S. Gukov, A. Mironov. Nucl. Phys. B. 1998. V. 517. P. 409.

[94] P. C. Argyres, A. D. Shapere. Nucl. Phys. B. 1996. V. 461. P. 437.

[95] N. Seiberg. Phys. Lett. B. 1996. V. 388. P. 753.

[96] K. Intriligator, D. R. Morrison, N. Seiberg. Nucl. Phys. B. 1997. V. 497. P. 56.

[97] O. Aharony, A. Hanany, B. Kol. Webs of $(p, q)$ branes, five-dimensional field theories and grid diagrams. JHEP, 9801, 002.

[98] A. Marshakov, A. Mironov. Nucl. Phys. B. 1998. V. 518. P. 59.

[99] A. Brandhuber, N. Itzhaki, J. Sonnenschain, S. Theisen, S. Yankielowicz. Phys. Lett. B. 1997. V. 415. P. 127.

[100] A. Gorsky. Phys. Lett. B. 1997. V. 410. P. 22.

[101] O. Aharony, A. Hanany. Branes, superpotentials and superconformal fixed points. hep-th/9704170.

[102] J. Barbon, A. Pasquinucci. Nucl. Phys. B. 1998. V. 517. P. 125; hep-th/9712135.

[103] P. Aspinwall, B. Greene, D. Morrison. Nucl. Phys. B. 1993. V. 416. P. 414.

[104] D.-E. Diaconescu. D-branes, monopoles and Nahm equation. hep-th/9608163.

[105] P. M. Sutcliffe. Phys. Lett. B. 1996. V. 381. P. 129.

[106] W. Nahm. The construction of all selfdual monopoles by ADHM method. In: Monopoles in Quantum Field Theories. Eds. N. Craigie, P. Goddard, W. Nahm. Singapore: World Scientific, 1982. P. 440.

[107] I. M. Krichever, D. H. Phong. On the integrable geometry of soliton equations and $N=2$ supersymmetric gauge theories. hep-th/9604199.

[108] A. Sen. Nucl. Phys. B. 1996. V. 475. P. 562.

[109] M. Douglas, M. Li. D-brane realization of super Yang-Mills theory in four dimensions. hep-th/9604041.

[110] М. Атья, Н. Хитчин. Геометрия и динамика магнитных монополей. М.: Мир, 1991.

[111] A. Sen. Phys. Rev. D. 1997. V. 55. P. 2501.

[112] G. Moore, E. Witten. Integration over $u$-plane in Donaldson theory. hep-th/9709193; A. Losev, N. Nekrasov, S. Shatashvili. Issues in topological gauge theories. hep-th/9711108; Testing Sieberg-Witten solution. hep-th/9801061; A. Gorsky, A. Mironov, A. Marshakov, A. Morozov. Nucl. Phys. B. 1998. V. 527. P. 690; K. Takasaki. Integrable hierarchies and contact terms in $u$-plane integrals of topologically twisted supersymmetric gauge theories. hep-th/9893217. 